\title{
Inheritance of Resistance to Powdery Mildew Race 1W in Watermelon
}

\author{
Yariv Ben-Naim and Yigal Cohen
}

Faculty of Life Sciences, Bar Ilan University, Ramat-Gan 52900, Israel.

Accepted for publication 24 July 2015.

\begin{abstract}
Ben-Naim, Y., and Cohen, Y. 2015. Inheritance of resistance to powdery mildew race $1 \mathrm{~W}$ in watermelon. Phytopathology 105:1446-1457.

Powdery mildew caused by Podosphaera xanthii is a major disease of watermelon in Israel. In this study, 291 accessions of Citrullus spp. were evaluated for resistance against $P$. xanthii race $1 \mathrm{~W}$. Only eight accessions exhibited high level of resistance. Inheritance of resistance against P. xanthii race $1 \mathrm{~W}$ was studied by crossing three resistant accession of Citrullus lanatus var. citroides BIU 119, PI 189225, or PI 482312 with the

cotyledon stage and the 4-leaf stage and in the field, at the 15-leaf stage. Resistance at the cotyledon stage was controlled by a single, partially dominant gene, whereas at the 4-leaf stage or the 15-leaf stage resistance was controlled by three complimentary, partially dominant genes. Crosses made among these resistant accessions revealed that BIU 119 and PI 189225 carry the same genes for resistance, whereas PI 482312 shares two out of three genes with both BIU 119 and PI 189225. A breeding line with high resistance level and good fruit qualities was developed from BIU $119 \times$ HA5500.
\end{abstract} susceptible cultivar 'Malali' or 'Sugar Baby'. Parents, F1, F2, and back cross progenies were evaluated for resistance in growth chambers at the
Additional keyword: cucurbits.
Watermelon (Citrullus lanatus (Thunb.) Matsum \& Nakai (=Citrullus vulgaris Schrad. ex. Eckl. \& Zeyh)) is an annual summer crop widely grown in warm regions and greatly appreciated for its large, sweet fruit. Recent archeological data suggest that watermelon was grown near the Nile River, Egypt as early as 3985 BC, before it was domesticated in West Africa (Zohary et al. 2012). Watermelon is a highly valuable fresh fruit in Israel, available 9 months a year. During the winter it is grown in the Arava Valley and fruits are marketed as early as March. In the coastal plain it is grown under plastic cover in the spring and in the open field during the summer.

C. lanatus is closely related and crossable with the diploid wild taxa C. colocynthis (L.) Schard., C. ecirrohsus Cogn., and C. rehmii De Winter. The wild taxon Praecitrullus fistulosus (Stocks) Pangalo. is not crossable with the taxa above (Levi et al. 2005, 2010).

Powdery mildew caused by Podosphaera xanthii (Castagne) Braun \& Shishkoff (syn. Sphaerotheca fuliginea (Schlechttend.:Fr.) Pollacci auct.p.p.) is a major disease of watermelon in Israel, attacking the crop at all seasons and all locations. Because no resistant cultivars are available, farmers rely mainly on fungicides to control the disease. Powdery mildew reduces the number of fruits per plant, fruit size, fruit quality, flavor, and storage life (Keinath and DuBose 2004; McGrath and Thomas 1996).

Golovinomyces cichoracearum (D.C.) V.P. Heluta (formerly Erysiphe cichoracearum D.C.) is another powdery mildew agent of cucurbits. In Ukraine (Tomason and Gibson 2006) and the Czech Republic (Lebeda et al. 2011) both G. cichoracearum and P. xanthii occur on watermelon. G. cichoracearum was not found on watermelon in Israel (Cohen and Eyal 1995), the United States, or Mexico (Felix-Gastelum et al. 2005).

A set of differential melon lines (Cucumis melo) enables the distinction between races of $P$. xanthii (McCreight et al. 1987; Pitrat et al. 1998). Kenigsbuch and Cohen (1992) reported that in melon,

Corresponding author: Y. Cohen; E-mail address: yigal.cohen1@gmail.com

http://dx.doi.org/10.1094/PHYTO-02-15-0048-R

(C) 2015 The American Phytopathological Society
$P m-1$ and $P m-3$ are responsible for resistance against race 1, while $P m-2, P m-4$, and $P m-6$ are responsible for resistance against race 2 .

Powdery mildew causes economic damage in watermelon crops in the United States since 1996 (Davis et al. 2001; Keinath and DuBose 2004; McGrath 2001). Recently, outbreaks of powdery mildew races $1 \mathrm{~W}, 2 \mathrm{~W}, 2 \mathrm{WU} . \mathrm{S}$, and $2 \mathrm{WF}$ were confirmed on watermelon in many U.S. states (Davis et al. 2007; Kousik et al. 2011; Mercier et al. 2014; Zhang et al. 2011).

Extensive efforts were done to identify resistance against powdery mildew in watermelon. Davis et al. (2001) screened 110 watermelon plant introduction (PI) for resistance to race $1 \mathrm{~W}$ of P. xanthii (Davis et al. 2001). They released PI 525088 as multigenic, highly resistant line to race 1W (Davis et al. 2006a). This resistance appeared to be independent in resistance to P. xanthii race 2W (Davis et al. 2002, 2006b; Thomas et al. 2005).

In a later study, eight accessions were found highly resistant among 1,573 accessions screened for resistance to race $1 \mathrm{~W}$ (Davis et al. 2007). Tetteh et al. (2010) found in the same population nine accessions highly resistant to race 2W: PI 189225 exhibited polygenetic inheritance of resistance (Tetteh et al. 2010) and PI 270545 carried recessive gene $p m r-1$ and dominant gene Pmr-2 for moderate resistance (Tetteh et al. 2013a). In a recent study they showed that resistance against race 2WU.S in a population derived from a cross between the resistant PI 189225 and the susceptible 'Charleston Gray' is controlled by a single dominant gene (Tetteh et al. 2013b). A screen for new sources of resistance to race $2 \mathrm{WF}$ in the United States revealed that most genotypes from Zimbabwe are highly resistant to this race (Zhang et al. 2011). Kim et al. (2013) reported that the Indian watermelon Arka Manik carries a single, partially dominant gene for resistance to powdery mildew race $1 \mathrm{~W}$.

In Israel, all commercial watermelon cultivars, diploid or triploid, are susceptible to powdery mildew. The objective of the current study was to develop watermelon lines resistant to $P$. xanthii race $1 \mathrm{~W}$, the most frequent race in Israel (Cohen et al. 2000). For this purpose we examined 291 accessions of Citrullus spp. for resistance to race $1 \mathrm{~W}$ of $P$. xanthii. We discovered new sources of resistance effective against this race of powdery mildew, determined the mode of inheritance in three resistant accessions and developed from one accession a high-quality resistant line. 
TABLE 1. Response to Podospheara xanthii race $1 \mathrm{~W}$ of entries 1 to 291 of watermelon accessions ${ }^{\mathrm{a}}$

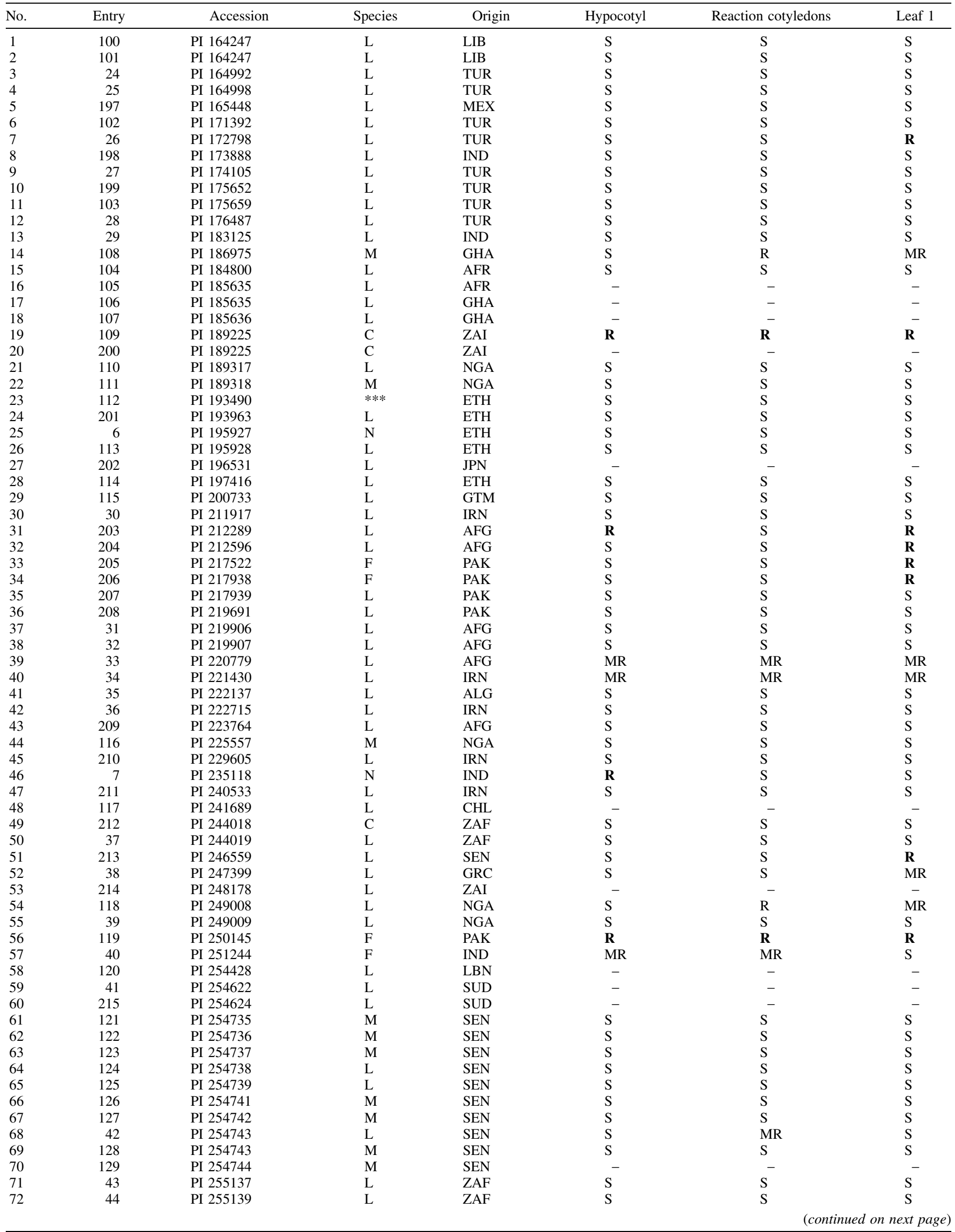

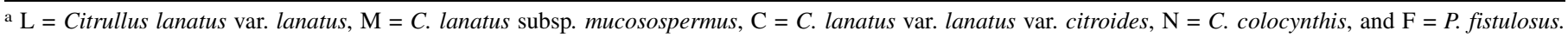
$*$ C e ecirrhosus, $* * C$. rehmii, and $* * * C$. dipsaceus (supplied as Citrullus). Bold $\mathrm{R}$ indicates resistance to powdery mildew race $1 \mathrm{~W}$. 
TABLE 1. (continued from preceding page)

\begin{tabular}{|c|c|c|c|c|c|c|c|}
\hline No. & Entry & Accession & Species & Origin & Hypocotyl & Reaction cotyledons & Leaf 1 \\
\hline 73 & 45 & PI 255662 & $\mathrm{~L}$ & AFG & $\mathrm{S}$ & $\mathrm{S}$ & $\mathrm{S}$ \\
\hline 74 & 216 & PI 260733 & $\mathrm{~L}$ & SUD & - & - & - \\
\hline 75 & 8 & PI 269365 & $\mathrm{~N}$ & AFG & $\mathrm{S}$ & $\mathrm{S}$ & $\mathrm{S}$ \\
\hline 76 & 88 & PI 269365 & $\mathrm{~N}$ & AFG & $\mathrm{S}$ & MR & MR \\
\hline 77 & 217 & PI 269464 & $\mathrm{~L}$ & PAK & - & - & - \\
\hline 78 & 46 & PI 270548 & $\mathrm{~L}$ & GHA & $\mathrm{S}$ & $\mathrm{S}$ & $\mathrm{S}$ \\
\hline 79 & 131 & PI 270549 & $\mathrm{~L}$ & GNA & $\mathrm{S}$ & $\mathrm{S}$ & $\mathrm{S}$ \\
\hline 80 & 132 & PI 270550 & $\mathrm{~L}$ & GNA & $\mathrm{S}$ & $\mathrm{S}$ & $\mathrm{S}$ \\
\hline 81 & 133 & PI 270551 & $\mathrm{~L}$ & GNA & $\mathrm{S}$ & $\mathrm{S}$ & $\mathrm{S}$ \\
\hline 82 & 218 & PI 270562 & $\mathrm{C}$ & $\mathrm{ZAF}$ & - & - & - \\
\hline 83 & 219 & PI 270564 & $\mathrm{C}$ & $\mathrm{ZAF}$ & - & - & - \\
\hline 84 & 220 & PI 270565 & $\mathrm{~L}$ & $\mathrm{ZAF}$ & $\mathrm{S}$ & $\mathrm{S}$ & $\mathrm{S}$ \\
\hline 85 & 134 & PI 271132 & 1 & TUN & $\mathrm{S}$ & $\mathrm{S}$ & $\mathrm{S}$ \\
\hline 86 & 135 & PI 271133 & 1. & TUN & $\mathrm{S}$ & $\mathrm{S}$ & $\mathrm{S}$ \\
\hline 87 & 136 & PI 271133 & $\mathrm{~L}$ & TUN & - & - & - \\
\hline 88 & 47 & PI 271750 & $\mathrm{~L}$ & GHA & $\mathrm{S}$ & $\mathrm{S}$ & $\mathrm{S}$ \\
\hline 89 & 48 & PI 271751 & $\mathrm{~L}$ & GHA & - & - & - \\
\hline 90 & 221 & PI 271752 & $\mathrm{~L}$ & GHA & - & - & - \\
\hline 91 & 262 & PI 271769 & $\mathrm{~L}$ & $\mathrm{ZAF}$ & $\mathrm{S}$ & $\mathrm{S}$ & $\mathrm{S}$ \\
\hline 92 & 49 & PI 271770 & $\mathrm{~L}$ & $\mathrm{ZAF}$ & $\mathrm{S}$ & $\mathrm{S}$ & $\mathrm{S}$ \\
\hline 93 & 222 & PI 271773 & $\mathrm{C}$ & $\mathrm{ZAF}$ & $\mathrm{S}$ & $\mathrm{S}$ & MR \\
\hline 94 & 50 & PI 271774 & $\mathrm{~L}$ & $\mathrm{ZAF}$ & $\mathbf{R}$ & $\mathrm{S}$ & $\mathrm{S}$ \\
\hline 95 & 137 & PI 271774 & $\mathrm{~L}$ & $\mathrm{ZAF}$ & - & - & - \\
\hline 96 & 51 & PI 271775 & $\mathrm{~L}$ & $\mathrm{ZAF}$ & MR & $\mathrm{S}$ & $\mathrm{S}$ \\
\hline 97 & 138 & PI 271776 & $\mathrm{~L}$ & $\mathrm{ZAF}$ & $\mathrm{S}$ & $\mathrm{S}$ & $\mathrm{S}$ \\
\hline 98 & 139 & PI 271777 & $\mathrm{~L}$ & $\mathrm{ZAF}$ & $\mathrm{S}$ & $\mathrm{S}$ & $\mathrm{S}$ \\
\hline 99 & 140 & PI 271778 & $\mathrm{~L}$ & $\mathrm{ZAF}$ & $\mathrm{S}$ & $\mathrm{S}$ & $\mathrm{S}$ \\
\hline 100 & 223 & PI 271981 & $\mathrm{~L}$ & SOM & $\mathrm{S}$ & $\mathrm{S}$ & MR \\
\hline 101 & 224 & PI 271982 & $\mathrm{~L}$ & SOM & - & - & - \\
\hline 102 & 225 & PI 271986 & $\mathrm{~L}$ & SOM & - & - & - \\
\hline 103 & 52 & PI 271988 & $\mathrm{~L}$ & SOM & - & - & - \\
\hline 104 & 53 & PI 273479 & $\mathrm{~L}$ & ETH & $\mathrm{S}$ & $\mathrm{S}$ & $\mathrm{S}$ \\
\hline 105 & 54 & PI 273480 & $\mathrm{~L}$ & ETH & $\mathrm{S}$ & $\mathrm{S}$ & $\mathrm{S}$ \\
\hline 106 & 55 & PI 273481 & $\mathrm{~L}$ & ETH & $\mathrm{S}$ & $\mathrm{S}$ & $\mathrm{S}$ \\
\hline 107 & 164 & PI 274035 & $\mathrm{~L}$ & $\mathrm{ZAF}$ & $\mathrm{S}$ & $\mathrm{S}$ & $\mathrm{S}$ \\
\hline 108 & 56 & PI 274794 & $\mathrm{~L}$ & PAK & $\mathbf{R}$ & $\mathrm{S}$ & $\mathbf{R}$ \\
\hline 109 & 57 & PI 276445 & $\mathrm{~L}$ & JOR & $\mathrm{S}$ & $\mathrm{S}$ & $\mathrm{S}$ \\
\hline 110 & 226 & PI 278005 & $\mathrm{~L}$ & TUR & $\mathrm{S}$ & $\mathrm{S}$ & $\mathrm{S}$ \\
\hline 111 & 58 & PI 278016 & $\mathrm{~L}$ & TUR & $\mathrm{S}$ & $\mathrm{S}$ & $\mathrm{S}$ \\
\hline 112 & 141 & PI 278045 & $\mathrm{~L}$ & TUR & $\mathrm{S}$ & $\mathrm{S}$ & $\mathrm{S}$ \\
\hline 113 & 142 & PI 278050 & $\mathrm{~L}$ & TUR & $\mathrm{S}$ & $\mathrm{S}$ & $\mathrm{S}$ \\
\hline 114 & 143 & PI 278061 & $\mathrm{~L}$ & TUR & $\mathrm{S}$ & $\mathrm{S}$ & $\mathrm{S}$ \\
\hline 115 & 227 & PI 279456 & $\mathrm{~L}$ & JPN & $\mathrm{S}$ & $\mathrm{S}$ & $\mathbf{R}$ \\
\hline 116 & 228 & PI 295842 & $\mathrm{C}$ & $\mathrm{ZAF}$ & $\mathrm{S}$ & $\mathrm{S}$ & $\mathbf{R}$ \\
\hline 117 & 144 & PI 295845 & I & $\mathrm{ZAF}$ & $\mathrm{S}$ & $\mathrm{S}$ & $\mathrm{S}$ \\
\hline 118 & 229 & PI 295848 & $\mathrm{~L}$ & ZAF & $\mathrm{S}$ & $\mathrm{S}$ & $\mathrm{S}$ \\
\hline 119 & 145 & PI 295850 & $\mathrm{C}$ & $\mathrm{ZAF}$ & $\mathrm{S}$ & $\mathrm{S}$ & $\mathrm{S}$ \\
\hline 120 & 79 & PI 296332 & $\mathrm{~L}$ & $\mathrm{ZAF}$ & $\mathrm{S}$ & $\mathrm{S}$ & $\mathrm{S}$ \\
\hline 121 & 80 & PI 296335 & $\mathrm{C}$ & $\mathrm{ZAF}$ & $\mathrm{S}$ & $\mathrm{S}$ & $\mathrm{S}$ \\
\hline 122 & 81 & PI 296337 & $\mathrm{C}$ & $\mathrm{ZAF}$ & $\mathrm{S}$ & $\mathrm{S}$ & $\mathrm{S}$ \\
\hline 123 & 78 & PI 296341 & $\mathrm{C}$ & $\mathrm{ZAF}$ & MR & $\mathrm{S}$ & $\mathrm{S}$ \\
\hline 124 & 165 & PI 296341 & $\mathrm{C}$ & $\mathrm{ZAF}$ & $\mathrm{S}$ & $\mathrm{S}$ & $\mathrm{S}$ \\
\hline 125 & 166 & PI 296341 & $\mathrm{C}$ & $\mathrm{ZAF}$ & $\mathrm{S}$ & $\mathrm{S}$ & $\mathrm{S}$ \\
\hline 126 & 167 & PI 296342 & $\mathrm{C}$ & $\mathrm{ZAF}$ & $\mathrm{S}$ & $\mathrm{S}$ & $\mathrm{S}$ \\
\hline 127 & 168 & PI 296342 & $\mathrm{C}$ & $\mathrm{ZAF}$ & $\mathrm{S}$ & $\mathrm{S}$ & $\mathrm{S}$ \\
\hline 128 & 169 & PI 296343 & $\mathrm{C}$ & $\mathrm{ZAF}$ & $\mathrm{S}$ & $\mathrm{S}$ & $\mathrm{S}$ \\
\hline 129 & 170 & PI 296343 & $\mathrm{C}$ & $\mathrm{ZAF}$ & - & - & - \\
\hline 130 & 130 & PI 296466 & $\mathrm{~L}$ & PAK & - & - & - \\
\hline 131 & 146 & PI 299378 & $\mathrm{C}$ & $\mathrm{ZAF}$ & $\mathrm{S}$ & $\mathrm{S}$ & $\mathrm{S}$ \\
\hline 132 & 59 & PI 306782 & $\mathrm{~L}$ & NGA & $\mathrm{S}$ & $\mathrm{S}$ & $\mathrm{S}$ \\
\hline 133 & 60 & PI 307608 & $\mathrm{~L}$ & NGA & $\mathrm{S}$ & $\mathrm{S}$ & $\mathrm{S}$ \\
\hline 134 & 230 & PI 307609 & $\mathrm{~L}$ & NGA & $\mathrm{S}$ & $\mathrm{S}$ & $\mathrm{S}$ \\
\hline 135 & 171 & PI 314655 & $\mathrm{~L}$ & UZB & $\mathrm{S}$ & $\mathrm{S}$ & $\mathrm{S}$ \\
\hline 136 & 61 & PI 319212 & $\mathrm{~L}$ & EGY & $\mathrm{S}$ & $\mathrm{S}$ & $\mathrm{S}$ \\
\hline 137 & 62 & PI 326515 & $\mathrm{~L}$ & GHA & $\mathrm{S}$ & $\mathrm{S}$ & $\mathrm{S}$ \\
\hline 138 & 147 & PI 331106 & $\mathrm{~L}$ & URU & $\mathrm{S}$ & $\mathrm{S}$ & $\mathrm{S}$ \\
\hline 139 & 232 & PI 371746 & $\mathrm{~F}$ & IND & $\mathrm{S}$ & $\mathrm{S}$ & MR \\
\hline 140 & 233 & PI 371747 & $\mathrm{~F}$ & IND & $\mathrm{S}$ & $\mathrm{S}$ & MR \\
\hline 141 & 234 & PI 371754 & $\mathrm{~F}$ & IND & $\mathrm{S}$ & $\mathrm{S}$ & $\mathbf{R}$ \\
\hline 142 & 148 & PI 378614 & $\mathrm{~L}$ & ZAI & $\mathrm{S}$ & $\mathrm{S}$ & $\mathrm{S}$ \\
\hline 143 & 149 & PI 378615 & $\mathrm{~L}$ & ZAI & $\mathrm{S}$ & $\mathrm{S}$ & $\mathrm{S}$ \\
\hline 144 & 231 & PI 381743 & $\mathrm{~F}$ & IND & $\mathrm{S}$ & $\mathrm{S}$ & MR \\
\hline 145 & 150 & PI 381748 & $\mathrm{~F}$ & IND & $\mathbf{R}$ & $\mathbf{R}$ & $\mathbf{R}$ \\
\hline \multirow[t]{2}{*}{146} & 63 & PI 381749 & $\mathrm{~F}$ & IND & $\mathbf{R}$ & \multirow{2}{*}{\multicolumn{2}{|c|}{ (continued on next page }} \\
\hline & & & & & & & \\
\hline
\end{tabular}


TABLE 1. (continued from preceding page)

\begin{tabular}{|c|c|c|c|c|c|c|c|}
\hline No. & Entry & Accession & Species & Origin & Hypocotyl & Reaction cotyledons & Leaf 1 \\
\hline 147 & 64 & PI 381750 & $\mathrm{~F}$ & IND & $\mathbf{R}$ & $\mathrm{S}$ & $\mathrm{S}$ \\
\hline 148 & 65 & PI 381752 & $\mathrm{~F}$ & IND & $\mathrm{S}$ & $\mathrm{S}$ & $\mathrm{S}$ \\
\hline 149 & 151 & PI 381755 & $\mathrm{~F}$ & IND & $\mathbf{R}$ & $\mathbf{R}$ & $\mathbf{R}$ \\
\hline 150 & 235 & PI 385963 & $\mathrm{~L}$ & KEN & $\mathrm{S}$ & $\mathrm{S}$ & $\mathrm{S}$ \\
\hline 151 & 236 & PI 385964 & $\mathrm{~L}$ & KEN & $\mathrm{S}$ & $\mathrm{S}$ & $\mathrm{S}$ \\
\hline 152 & 89 & PI 386014 & $\mathrm{~N}$ & USA & $\mathrm{S}$ & MR & MR \\
\hline 153 & 90 & PI 386015 & $\mathrm{~N}$ & IRN & $\mathrm{S}$ & MR & MR \\
\hline 154 & 91 & PI 386016 & $\mathrm{~N}$ & IRN & $\mathrm{S}$ & MR & MR \\
\hline 155 & 9 & PI 386018 & $\mathrm{~N}$ & IRN & $\mathrm{S}$ & $\mathrm{S}$ & - \\
\hline 156 & 92 & PI 386019 & $\mathrm{~N}$ & IRN & $\mathrm{S}$ & MR & MR \\
\hline 157 & 93 & PI 386024 & $\mathrm{~N}$ & IRN & $\mathrm{S}$ & MR & MR \\
\hline 158 & 10 & PI 386026 & $\mathrm{~N}$ & IRN & $\mathrm{S}$ & $\mathrm{S}$ & $\mathrm{S}$ \\
\hline 159 & 94 & PI 386026 & $\mathrm{~N}$ & IRN & $\mathrm{S}$ & MR & MR \\
\hline 160 & 1 & PI 388770 & $\mathrm{~N}$ & MAR & MR & MR & $\mathrm{S}$ \\
\hline 161 & 237 & PI 427298 & $\mathrm{~F}$ & IND & $\mathrm{S}$ & $\mathrm{S}$ & $\mathrm{R}$ \\
\hline 162 & 95 & PI 432377 & $\mathrm{~N}$ & CYP & $\mathrm{S}$ & MR & MR \\
\hline 163 & 152 & PI 438675 & $\mathrm{~L}$ & MEX & $\mathrm{S}$ & $\mathrm{S}$ & $\mathrm{S}$ \\
\hline 164 & 153 & PI 442826 & $\mathrm{~L}$ & BRA & $\mathrm{S}$ & $\mathrm{S}$ & $\mathrm{S}$ \\
\hline 165 & 66 & PI 457916 & M & LIB & $\mathbf{R}$ & $\mathbf{R}$ & $\mathrm{S}$ \\
\hline 166 & 238 & PI 482283 & $\mathrm{C}$ & ZIM & $\mathrm{S}$ & $\mathrm{S}$ & $\mathrm{S}$ \\
\hline 167 & 239 & PI 482293 & $\mathrm{C}$ & ZIM & $\mathrm{S}$ & $\mathrm{S}$ & $\mathrm{S}$ \\
\hline 168 & 240 & PI 482298 & $\mathrm{C}$ & ZIM & $\mathrm{S}$ & $\mathrm{S}$ & MR \\
\hline 169 & 67 & PI 482299 & $\mathrm{C}$ & ZIM & MR & MR & MR \\
\hline 170 & 68 & PI 482300 & $\mathrm{C}$ & ZIM & MR & MR & MR \\
\hline 171 & 69 & PI 482301 & $\mathrm{C}$ & ZIM & MR & MR & $\mathrm{S}$ \\
\hline 172 & 154 & PI 482312 & $\mathrm{C}$ & ZIM & $\mathbf{R}$ & $\mathbf{R}$ & $\mathbf{R}$ \\
\hline 173 & 155 & PI 482315 & $\mathrm{C}$ & ZIM & $\mathrm{S}$ & $\mathrm{S}$ & $\mathrm{S}$ \\
\hline 174 & 156 & PI 482316 & $\mathrm{C}$ & ZIM & $\mathrm{S}$ & $\mathrm{S}$ & $\mathrm{S}$ \\
\hline 175 & 70 & PI 482319 & $\mathrm{~L}$ & ZIM & MR & MR & $\mathrm{S}$ \\
\hline 176 & 71 & PI 482321 & $\mathrm{~L}$ & ZIM & MR & MR & MR \\
\hline 177 & 241 & PI 482326 & $\mathrm{C}$ & ZIM & $\mathrm{S}$ & $\mathrm{S}$ & MR \\
\hline 178 & 242 & PI 482335 & $\mathrm{C}$ & ZIM & $\mathrm{S}$ & $\mathrm{S}$ & $\mathbf{R}$ \\
\hline 179 & 243 & PI 482336 & $\mathrm{C}$ & ZIM & $\mathrm{S}$ & $\mathrm{S}$ & $\mathrm{S}$ \\
\hline 180 & 82 & PI 490377 & $\mathrm{~L}$ & MLI & $\mathrm{S}$ & $\mathrm{S}$ & $\mathrm{S}$ \\
\hline 181 & 172 & PI 480378 & M & MLI & $\mathrm{S}$ & $\mathrm{S}$ & $\mathrm{S}$ \\
\hline 182 & 83 & PI 490379 & $\mathrm{~L}$ & MLI & $\mathrm{S}$ & $\mathrm{S}$ & $\mathrm{S}$ \\
\hline 183 & 173 & PI 490380 & $\mathrm{~L}$ & MLI & $\mathrm{S}$ & $\mathrm{S}$ & $\mathrm{S}$ \\
\hline 184 & 174 & PI 490381 & $\mathrm{M}$ & MLI & $\mathrm{S}$ & $\mathrm{S}$ & $\mathrm{S}$ \\
\hline 185 & 252 & PI 490382 & M & MLI & $\mathrm{S}$ & $\mathrm{S}$ & $\mathrm{S}$ \\
\hline 186 & 175 & PI 490384 & $\mathrm{M}$ & MLI & $\mathrm{S}$ & $\mathrm{S}$ & $\mathrm{S}$ \\
\hline 187 & 84 & PI 490385 & $\mathrm{~L}$ & MLI & $\mathrm{S}$ & $\mathrm{S}$ & $\mathrm{S}$ \\
\hline 188 & 176 & PI 490386 & $\mathrm{M}$ & MLI & $\mathrm{S}$ & $\mathrm{S}$ & $\mathrm{S}$ \\
\hline 189 & 244 & PI 500303 & $\mathrm{~L}$ & ZAM & - & - & - \\
\hline 190 & 72 & PI 500355 & $\mathrm{C}$ & ZAM & MR & MR & MR \\
\hline 191 & 157 & PI 505604 & $\mathrm{C}$ & ZAM & $\mathrm{S}$ & $\mathrm{S}$ & $\mathrm{S}$ \\
\hline 192 & 254 & PI 525088 & $\mathrm{~L}$ & EGY & $\mathrm{S}$ & $\mathrm{S}$ & MR \\
\hline 193 & 73 & TB81/464 & $\mathrm{L}$ & NGA & MR & $\mathrm{S}$ & $\mathrm{S}$ \\
\hline 194 & 74 & TB81/467 & $\bar{L}$ & NGA & $\mathrm{S}$ & $\mathrm{S}$ & $\mathrm{S}$ \\
\hline 195 & 75 & TB81/472 & $\mathrm{L}$ & NGA & MR & MR & MR \\
\hline 196 & 158 & TB81/473 & $\mathrm{L}$ & NGA & $\mathrm{S}$ & $\mathrm{S}$ & S \\
\hline 197 & 159 & TB81/474 & $\mathrm{L}$ & NGA & $\mathrm{S}$ & $\mathrm{S}$ & $\mathrm{S}$ \\
\hline 198 & 160 & TB81/476 & $\mathrm{L}$ & NGA & $\mathrm{S}$ & $\mathrm{S}$ & $\mathrm{S}$ \\
\hline 199 & 161 & TB81/477 & $\bar{L}$ & NGA & $\mathrm{S}$ & $\mathrm{S}$ & $\mathrm{S}$ \\
\hline 200 & 247 & TB81/485 & $\mathrm{L}$ & NGA & $\mathrm{S}$ & $\mathrm{S}$ & MR \\
\hline 201 & 248 & TB81/486 & $\mathrm{L}$ & NGA & $\mathrm{S}$ & $\mathrm{S}$ & MR \\
\hline 202 & 249 & TB81/514 & $\mathrm{L}$ & NGA & $\mathrm{S}$ & $\mathrm{S}$ & MR \\
\hline 203 & 250 & TB81/575 & $\bar{L}$ & NGA & - & - & - \\
\hline 204 & 96 & TB81/728 & $\mathrm{N}$ & NGA & - & - & - \\
\hline 205 & 97 & TE81/185 & $\mathrm{N}$ & NGA & $\mathrm{S}$ & $\mathbf{R}$ & $\mathbf{R}$ \\
\hline 206 & 98 & TE81/192 & $\mathrm{N}$ & NGA & $\mathrm{S}$ & $\mathbf{R}$ & $\mathbf{R}$ \\
\hline 207 & 19 & TE81/193 & $\mathrm{N}$ & NGA & $\mathrm{S}$ & $\mathrm{S}$ & $\mathrm{S}$ \\
\hline 208 & 99 & TE81/194 & $\mathrm{N}$ & NGA & $\mathrm{S}$ & $\mathrm{S}$ & $\mathrm{S}$ \\
\hline 209 & 20 & TE81/195 & $\mathrm{N}$ & NGA & $\mathrm{S}$ & $\mathrm{S}$ & $\mathrm{S}$ \\
\hline 210 & 277 & $\mathrm{CC}-2$ & $\mathrm{~N}$ & EGY & $\mathrm{S}$ & $\mathrm{S}$ & $\mathrm{S}$ \\
\hline 211 & 286 & $\mathrm{CC}-3$ & $\mathrm{~N}$ & EGY & $\mathrm{S}$ & $\mathrm{S}$ & $\mathrm{S}$ \\
\hline 212 & 287 & CC-5 & $\mathrm{N}$ & ISR & $\mathrm{S}$ & $\mathrm{S}$ & $\mathrm{S}$ \\
\hline 213 & 288 & CC-7 & $\mathrm{N}$ & EGY & $\mathrm{S}$ & $\mathrm{S}$ & $\mathrm{S}$ \\
\hline 214 & 289 & CC-8 & $\mathrm{N}$ & EGY & $\mathrm{S}$ & $\mathrm{S}$ & $\mathrm{S}$ \\
\hline 215 & 290 & CC-9 & $\mathrm{N}$ & ISR & $\mathrm{S}$ & $\mathrm{S}$ & $\mathrm{S}$ \\
\hline 216 & 18 & CC-9 & $\mathrm{N}$ & ISR & $\mathbf{R}$ & $\mathrm{S}$ & $\mathrm{S}$ \\
\hline 217 & 273 & CC-10 & $\mathrm{N}$ & ISR & $\mathrm{S}$ & $\mathrm{S}$ & $\mathrm{S}$ \\
\hline 218 & 12 & CC-11 & $\mathrm{N}$ & ISR & $\mathbf{R}$ & $\mathrm{S}$ & $\mathrm{S}$ \\
\hline 219 & 13 & CC-14 & $\mathrm{N}$ & ISR & $\mathbf{R}$ & $\mathrm{S}$ & $\mathrm{S}$ \\
\hline \multirow[t]{2}{*}{220} & 14 & CC-16 & $\mathrm{N}$ & ISR & $\mathbf{R}$ & $\mathbf{R}$ & $\mathbf{R}$ \\
\hline & & & & & & & (continued on next page) \\
\hline
\end{tabular}


TABLE 1. (continued from preceding page)

\begin{tabular}{|c|c|c|c|c|c|c|c|}
\hline No. & Entry & Accession & Species & Origin & Hypocotyl & Reaction cotyledons & Leaf 1 \\
\hline 221 & 274 & CC-16 & $\mathrm{N}$ & ISR & $\mathrm{S}$ & $\mathrm{S}$ & $\mathrm{S}$ \\
\hline 222 & 15 & $\mathrm{CC}-17$ & $\mathrm{~N}$ & ISR & $\mathbf{R}$ & $\mathrm{S}$ & $\mathbf{R}$ \\
\hline 223 & 275 & CC-17 & $\mathrm{N}$ & ISR & $\mathrm{S}$ & $\mathrm{S}$ & $\mathrm{S}$ \\
\hline 224 & 276 & CC-19 & $\mathrm{N}$ & ISR & $\mathrm{S}$ & $\mathrm{S}$ & $\mathrm{S}$ \\
\hline 225 & 278 & CC-20 & $\mathrm{N}$ & ISR & $\mathrm{S}$ & $\mathrm{S}$ & $\mathrm{S}$ \\
\hline 226 & 279 & CC-21 & $\mathrm{N}$ & ISR & $\mathrm{S}$ & $\mathrm{S}$ & $\mathrm{S}$ \\
\hline 227 & 280 & CC-22 & $\mathrm{N}$ & ISR & $\mathrm{S}$ & $\mathrm{S}$ & $\mathrm{S}$ \\
\hline 228 & 281 & CC-23 & $\mathrm{N}$ & ISR & $\mathrm{S}$ & $\mathrm{S}$ & $\mathrm{S}$ \\
\hline 229 & 282 & CC-24 & $\mathrm{N}$ & ISR & $\mathrm{S}$ & $\mathrm{S}$ & $\mathrm{S}$ \\
\hline 230 & 16 & CC-25 & $\mathrm{N}$ & ISR & $\mathbf{R}$ & $\mathbf{R}$ & $\mathrm{S}$ \\
\hline 231 & 17 & CC-27 & $\mathrm{N}$ & ISR & $\mathbf{R}$ & $\mathrm{S}$ & $\mathrm{S}$ \\
\hline 232 & 283 & $\mathrm{CC}-27$ & $\mathrm{~N}$ & ISR & $\mathrm{S}$ & $\mathrm{S}$ & $\mathrm{S}$ \\
\hline 233 & 284 & CC-28 & $\mathrm{N}$ & ISR & $\mathrm{S}$ & $\mathrm{S}$ & $\mathrm{S}$ \\
\hline 234 & 285 & CC-29 & $\mathrm{N}$ & ISR & $\mathrm{S}$ & $\mathrm{S}$ & $\mathrm{S}$ \\
\hline 235 & 183 & CIT 115/1978 & $\mathrm{C}$ & $\mathrm{CHN}$ & $\mathrm{S}$ & $\mathrm{S}$ & $\mathrm{S}$ \\
\hline 236 & 184 & CIT 116/1978 & $\mathrm{C}$ & $\mathrm{CHN}$ & - & - & - \\
\hline 237 & 185 & CIT 119/1980 & $\mathrm{C}$ & PRT & $\mathrm{S}$ & $\mathrm{S}$ & $\mathrm{S}$ \\
\hline 238 & 195 & CIT 121/1977 & $\mathrm{C}$ & $\mathrm{ZAF}$ & $\mathrm{S}$ & $\mathrm{S}$ & $\mathrm{S}$ \\
\hline 239 & 22 & CIT 127/1970 & $\mathrm{C}$ & ZAF & $\mathrm{S}$ & $\mathrm{S}$ & $\mathrm{S}$ \\
\hline 240 & 87 & CIT 141/1975 & $\mathrm{N}$ & SEN & $\mathrm{S}$ & MR & MR \\
\hline 241 & 186 & CIT 35/1976 & $\mathrm{C}$ & BRA & $\mathrm{S}$ & $\mathrm{S}$ & $\mathrm{S}$ \\
\hline 242 & 187 & CIT 63/1975 & $\mathrm{C}$ & ITA & $\mathrm{S}$ & $\mathrm{S}$ & $\mathrm{S}$ \\
\hline 243 & 188 & CIT 65/1976 & $\mathrm{C}$ & ITA & $\mathrm{S}$ & $\mathrm{S}$ & $\mathrm{S}$ \\
\hline 244 & 189 & CIT 68/1975 & $\mathrm{C}$ & ITA & $\mathrm{S}$ & $\mathrm{S}$ & $\mathrm{S}$ \\
\hline 245 & 190 & TETRA 1 & $\mathrm{~L}$ & USA & $\mathrm{S}$ & $\mathrm{S}$ & $\mathrm{S}$ \\
\hline 246 & 191 & TETRA 2 & $\mathrm{~L}$ & USA & $\mathrm{S}$ & $\mathrm{S}$ & $\mathrm{S}$ \\
\hline 247 & 192 & TETRA 3 & $\mathrm{~L}$ & USA & $\mathrm{S}$ & $\mathrm{S}$ & $\mathrm{S}$ \\
\hline 248 & 193 & TETRA 4 & $\mathrm{~L}$ & USA & $\mathrm{S}$ & $\mathrm{S}$ & $\mathrm{S}$ \\
\hline 249 & 258 & Crimson sweet & $\mathrm{L}$ & ISR & $\mathrm{S}$ & $\mathrm{S}$ & $\mathrm{S}$ \\
\hline 250 & 253 & G200 & $\mathrm{L}$ & USA & - & - & - \\
\hline 251 & 267 & HA 1032 & $\mathrm{~L}$ & ISR & $S$ & $\mathrm{~S}$ & $\mathrm{~S}$ \\
\hline 252 & 265 & HA 6008 & $\mathrm{~L}$ & ISR & $\mathrm{S}$ & $\mathrm{S}$ & $\mathrm{S}$ \\
\hline 253 & 268 & HA 1042 & $\mathrm{~L}$ & ISR & $\mathrm{S}$ & $\mathrm{S}$ & $\mathrm{S}$ \\
\hline 254 & 266 & HA 5603 & $\mathrm{~L}$ & ISR & $\mathrm{S}$ & $\mathrm{S}$ & $\mathrm{S}$ \\
\hline 255 & 269 & HAZERA-8 & $\mathrm{L}$ & ISR & $\mathrm{S}$ & $\mathrm{S}$ & $\mathrm{S}$ \\
\hline 256 & 196 & King of heart & $\mathrm{L}$ & USA & $\mathrm{S}$ & $\mathrm{S}$ & $\mathrm{S}$ \\
\hline 257 & 162 & L. W 1298 & M & AFR & $\mathbf{R}$ & $\mathbf{R}$ & $\mathbf{R}$ \\
\hline 258 & 23 & L. WILD & $*$ & $\mathrm{ZAF}$ & $\mathrm{S}$ & $\mathrm{S}$ & $\mathrm{S}$ \\
\hline 259 & 4 & LOT $88-151$ & $\mathrm{~N}$ & EGY & $\mathrm{S}$ & $\mathrm{S}$ & $\mathrm{S}$ \\
\hline 260 & 5 & M-1411 & $\mathrm{N}$ & ISR & $\mathrm{S}$ & $\mathrm{S}$ & $\mathrm{S}$ \\
\hline 261 & 271 & M-1411 & $\mathrm{N}$ & ISR & $\mathrm{S}$ & $\mathrm{S}$ & $\mathrm{S}$ \\
\hline 262 & 272 & M-1535 & $\mathrm{N}$ & ISR & $\mathrm{S}$ & $\mathrm{S}$ & $\mathrm{S}$ \\
\hline 263 & 256 & Malali & $\mathrm{L}$ & ISR & $\mathrm{S}$ & $\mathrm{S}$ & $\mathrm{S}$ \\
\hline 264 & 255 & Mini Pool & $\mathrm{L}$ & ISR & $\mathrm{S}$ & $\mathrm{S}$ & $\mathrm{S}$ \\
\hline 265 & 11 & Plot 372 & $\mathrm{~N}$ & NGA & $\mathbf{R}$ & $\mathbf{R}$ & $\mathrm{S}$ \\
\hline 266 & 245 & Quality Tri & $\mathrm{L}$ & TWN & $\mathrm{S}$ & $\mathrm{S}$ & MR \\
\hline 267 & 246 & Qween of hearts & $\mathrm{L}$ & USA & - & - & - \\
\hline 268 & 257 & Sugar Baby & $\mathrm{L}$ & ISR & $\mathrm{S}$ & $\mathrm{S}$ & $\mathrm{S}$ \\
\hline 269 & 261 & Summit & $\mathrm{L}$ & ISR & $\mathrm{S}$ & $\mathrm{S}$ & $\mathrm{S}$ \\
\hline 270 & 21 & WIR-4339 & $\mathrm{N}$ & SUN & $\mathbf{R}$ & $\mathrm{S}$ & - \\
\hline 271 & 76 & 1 wir-3706 & $\mathrm{C}$ & SUM & $\mathrm{S}$ & $\mathrm{S}$ & $\mathrm{S}$ \\
\hline 272 & 85 & $69 / 1978$ & $\mathrm{~L}$ & ITA & - & - & - \\
\hline 273 & 194 & $71 / 352$ & $\mathrm{~L}$ & $\mathrm{ZAF}$ & $\mathrm{S}$ & $\mathrm{S}$ & $\mathrm{R}$ \\
\hline 274 & 77 & 77046 J. 14 & $* *$ & $\mathrm{ZAF}$ & - & - & - \\
\hline 275 & 163 & 77125 N. 16 & $\mathrm{~L}$ & ZAF & $\mathrm{S}$ & $\mathrm{S}$ & $\mathrm{S}$ \\
\hline 276 & 3 & ACC.62.023 & $\mathrm{N}$ & ITA & $\mathbf{R}$ & $\mathrm{S}$ & $\mathrm{S}$ \\
\hline 277 & 259 & Calhoun Gray & $\mathrm{L}$ & ISR & $\mathrm{S}$ & $\mathrm{S}$ & $\mathrm{S}$ \\
\hline 278 & 264 & China $6 / 2 / 3$ & $\mathrm{~L}$ & ISR & $\mathrm{S}$ & $\mathrm{S}$ & $\mathrm{S}$ \\
\hline 279 & 260 & Charelston Gray & $\mathrm{L}$ & ISR & $\mathrm{S}$ & $\mathrm{S}$ & $\mathrm{S}$ \\
\hline 280 & 86 & 234 & $\mathrm{~N}$ & HUN & $\mathrm{S}$ & $\mathrm{S}$ & $\mathrm{S}$ \\
\hline 281 & 2 & 284 & $\mathrm{~N}$ & EYG & $\mathbf{R}$ & MR & MR \\
\hline 282 & 270 & 284 & $\mathrm{~N}$ & ISR & $\mathrm{S}$ & $\mathrm{S}$ & $\mathrm{S}$ \\
\hline 283 & 263 & HA 5500 & $\mathrm{~L}$ & ISR & $\mathrm{S}$ & $\mathrm{S}$ & $\mathrm{S}$ \\
\hline 284 & 177 & 32776 & $\mathrm{~L}$ & TUR & - & - & - \\
\hline 285 & 178 & 33344 & $\mathrm{~L}$ & TUR & $\mathrm{S}$ & $\mathrm{S}$ & $\mathrm{S}$ \\
\hline 286 & 179 & 37073 & $\mathrm{~L}$ & TUR & $\mathrm{S}$ & $\mathrm{S}$ & $\mathrm{S}$ \\
\hline 287 & 180 & 38225 & $\mathrm{~L}$ & TUR & $\mathrm{S}$ & $\mathrm{S}$ & $\mathrm{S}$ \\
\hline 288 & 181 & 38480 & $\mathrm{~L}$ & TUR & $\mathrm{S}$ & $\mathrm{S}$ & $\mathrm{S}$ \\
\hline 289 & 182 & 38940 & $\mathrm{~L}$ & TUR & $\mathrm{S}$ & $\mathrm{S}$ & $\mathrm{S}$ \\
\hline 290 & 251 & 83508 & $\mathrm{~L}$ & $\mathrm{ZAF}$ & $\mathrm{S}$ & $\mathrm{S}$ & $\mathrm{S}$ \\
\hline 291 & 291 & BIU 119 & $\mathrm{C}$ & Unknown & $\mathbf{R}$ & $\mathbf{R}$ & $\mathbf{R}$ \\
\hline
\end{tabular}


In a preliminary study, we reported that among 250 accessions of C. lanatus and C. colocynthis, two entries were resistant to P. xanthii race $1 \mathrm{~W}$ (Cohen et al. 2000).

\section{MATERIALS AND METHODS}

Plants. A total of 291 Citrullus spp. and Parecitrulus fistulosus accessions were obtained in 1998 from the USDA via the Israel Gene Bank. The accessions, originating from 44 countries, included six main species/subspecies: C. lanatus var. lanatus (including subsp. mucosospermus); C. lanatus var. citroides; C. colocynthis; C. rehmii; C. ecirrhosus; and, P. fistulosus. Plants were grown from seeds in Speedling trays $(1 \times 1$ inch filled with 2:1 peat:vermiculite, $\mathrm{vol} / \mathrm{vol})$ in the greenhouse $\left(23\right.$ to $\left.30^{\circ} \mathrm{C}\right)$. About six to eight seeds of each accession were seeded. After germination plants were fertilized once a week with $1 \% \mathrm{~N}$ : P: K solution.

Pathogen and inoculation. Conidia were collected from powdery mildew-infected watermelon plants in the field. Microscopic examination of spore germination confirms it as $P$. xanthii.

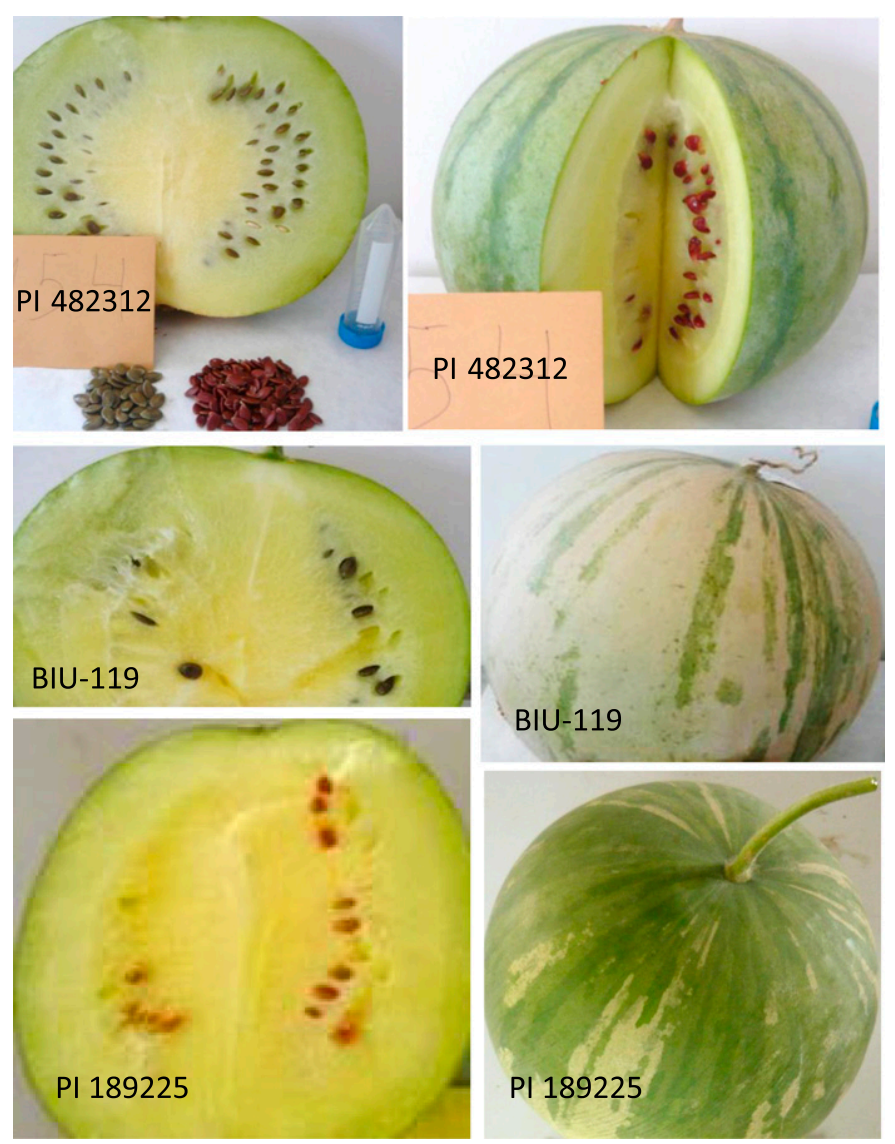

Fig. 1. Mature fruits of the three resistant watermelon accessions used in the present study.
The following melon (Cucumis melo) differential cultivars were included in all tests to determine the race identity of $P$. xanthii: 'Dulche', 'Hemed', 'Anannas-Yokneam' (AY), 'WNR-18', 'NoyIzrael', PI 124111, PI 124112, 'Ein Dor' (='PMR 45'), 'PMR 5', and 'PMR 6' (Kenigsbuch and Cohen 1992; McCreight et al. 1987; Pitrat et al. 1998). Accessions were evaluated for resistance to powdery mildew race $1 \mathrm{~W}$ at the cotyledon stage during 1999 (Cohen et al. 2000); the resistant accessions were propagated by self-pollination and re-examined for resistance to race $1 \mathrm{~W}$ during 2008 to 2012. Inoculation at the cotyledon stage and the 4-leaf stage was done in the greenhouse by dusting fresh conidia from infected susceptible Malali plants over the test plants $(\sim 150$ conidia $\left./ \mathrm{cm}^{2}\right)$. The resistant plants were selected and planted (April or August) in the field in a net-house $(6 \times 50 \mathrm{~m})$. Spreader plants (melon AY and watermelon Malali) infected with $P$. xanthii race $1 \mathrm{~W}$ were planted in the net-house to facilitate infection of the selected watermelon plants. Once a week, plants were blown with a back-pack mechanical blower to enhance conidial dispersal in the house.

Resistance to powdery mildew in original accessions. Inoculation was done at the cotyledon stage and disease was recorded at 10 days postinoculation (dpi) from the hypocotyl, cotyledons, and leaf one. The following visual categorical scale was used to rate the reaction to infection: $\mathrm{R}=$ resistant, no fungal colonies were seen; $\mathrm{MR}=$ moderately resistant, a few colonies with sparse sporulation were visible; $\mathrm{S}=$ susceptible, abundant colonies with profuse sporulation were visible. Thirty-four accessions (out of the 291) did not germinate or experienced early death of seedlings.

In some experiments, resistance/susceptibility was evaluated by counting the number of conidia produced by $P$. xanthii per unit leaf area. For spore yield determination, two leaf discs $(14 \mathrm{~mm}$ diameter) were removed from each leaf, placed in $2 \mathrm{ml}$ of water containing $0.1 \%$ Tween-20, and agitated for $10 \mathrm{~min}$, and the number of conidia was counted with the aid of a cytometer.

Resistance to powdery mildew in progeny plants. Parents, F1, F2, and back cross (BC) progeny plants were grown in Speedling trays and inoculated at the cotyledon stage or the 4-leaf stage by dusting fresh conidia as described above. Evaluation of disease development was done at $10 \mathrm{dpi}$ by using the following 0 to 4 visual scale: $0=$ no disease; $0.1,0.2 \ldots 0.9=10,20 \ldots .90 \%$ of the plant surface exhibit chlorotic or necrotic symptoms with sparse mycelium lacking conidia; 1 to $1.9=5$ to $25 \%$ of the plant surface is occupied with discrete sporulating fungal colonies, with or without chlorosis; 2 to $2.9=25$ to $50 \%$ of the plant surface is occupied with coalescing, sporulating fungal colonies; and 3 to $4=51$ to $100 \%$ of the plant surface is occupied with coalesced, heavily sporulating fungal colonies. The same visual scale was used to also evaluate the disease in mature plants in the field.

\section{RESULTS}

Disease resistance in original accessions. Table 1 presents the response of 291 accessions of Citrullus spp. to powdery mildew

TABLE 2. Phenotypic characteristics of three watermelon accessions resistant to powdery mildew $1 \mathrm{~W}$ used in this study

\begin{tabular}{|c|c|c|c|}
\hline \multirow[b]{2}{*}{ Character } & \multicolumn{3}{|c|}{ Accession } \\
\hline & BIU 119 & PI 189225 & PI 482312 \\
\hline Fruit shape & Flat round & Round & Flat round \\
\hline Fruit weight, kg & $12-21$ & $6-7$ & $10-16$ \\
\hline Flesh color & Yellow & Yellow & Yellow \\
\hline Rind thickness, cm & $3-3.5$ & $2.5-3$ & $3-3.5$ \\
\hline Seed color & Khaki & Red & Green or red \\
\hline Flowering & Andromonoecious & Monoecious & Andromonoecious \\
\hline Days to maturity & 120 & 110 & 120 \\
\hline Total soluble solids, $\%$ & 3.5 & 3 & 3.5 \\
\hline
\end{tabular}


incited by $P$. xanthi race $1 \mathrm{~W}$. Most entries were susceptible, classified as SSS showing colonies of powdery mildew on hypocotyl, cotyledons, and first true leaf. A few entries were classified SSR, SRR, RSS, RSR, or RRS (Table 1). High level of resistance, RRR, was expressed by three accessions of $C$. lanatus var. citroides, three accessions of Praecitrullus fistulosus, one accession of $C$. colocynthis, and one accession of $C$. lanatus subsp. mucosospermus. PI 525088 (reported resistant by Davis et al. 2006a) was susceptible.

Further experiments were conducted with only the three resistant accessions of $C$. lanatus var. citroides: BIU 119, PI 189225, and PI 482312. Their fruits are shown in Figure 1 and their phenotypic features are given in Table 2.

Quantitative assays for disease resistance. BIU 119, PI 189225, and PI 482312 developed significantly less disease (Fig. 2A) and allowed for a significantly reduced sporulation (Fig. 2B) compared with the commercial watermelon cultivars HA5500, Crimson Sweet, Malali, or Sugar Baby.

The response of BIU 119, PI 189225, PI 482312, and Malali to inoculation with increasing inoculum doses of $P$. xanthii is shown in Figure 3. The susceptible Malali produced heavy disease in hypocotyls, cotyledons, and first true leaf when inoculated with a low dose of $\sim 33$ spores $/ \mathrm{cm}^{2}$. Much lower disease levels compared with Malali were seen in all three resistant accessions at all five inoculum doses tested.

Inheritance of resistance. Crosses were made between each resistant accession and the susceptible cultivars Malali or Sugar Baby. The response to powdery mildew of the parents and their F1 progeny plants in the field is shown in Figure 4A. F1 plants of all crosses were moderately resistant. The relationship between leaf position and disease severity in field-grown 15-leaf parental and F1 plants of the cross Malali $\times$ PI 189225 is shown in Figure 4B. Disease severity on leaves of the resistant parent did not exceed a value of 0.5 compared with 1.0 to 3.1 in the susceptible parent. F1 plants showed values ranging between 0.5 and 2 . The two oldest and two youngest leaves showed less disease compared with middle leaves (Fig. 4B).

Data on the inheritance of disease resistance are given in Tables 3, 4 , and 5. They show the segregation of powdery mildew resistance in the offspring plant populations obtained from three crosses made between each resistant accession and a susceptible commercial cultivar. Response to the disease was estimated in F1, F2, and BC plants at the cotyledon stage, 4-leaf stage, and 15-leaf stage plants in the field.

Resistance at the cotyledon stage (Table 3) was controlled by a single, partially dominant gene in all three resistant accessions. The F2 progeny plants segregated R/MR/S (resistant; moderately resistant; susceptible) at a ratio of $1 / 2 / 1$, the $\mathrm{BCr}$ at $1 / 1 / 0$ and the BCs at $0 / 1 / 1$.

A different mode of inheritance was observed at the 4-leaf stage (Table 4). F1 plants were moderately resistant and the F2 population segregated $\mathrm{R} / \mathrm{MR} / \mathrm{S}$ at a ratio of $7 / 37 / 20$, suggesting that resistance is controlled by the complementary (additive) action of five or six partially dominant alleles donated by three genes. Plants showing the MR response probably carried three or four partially dominant alleles.

The mode of inheritance in adult plants in the field was slightly different from the previous model. F2 populations segregated

A

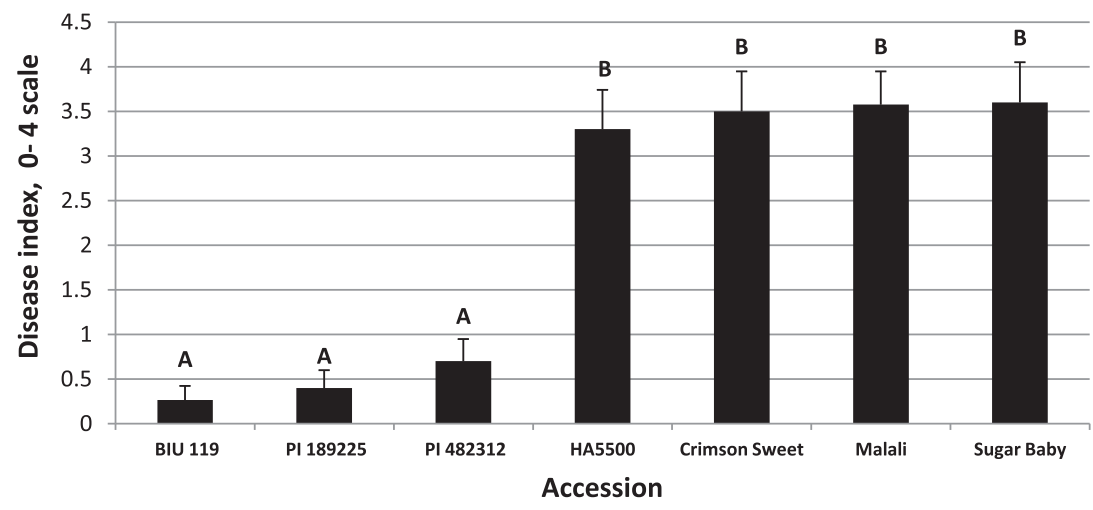

B

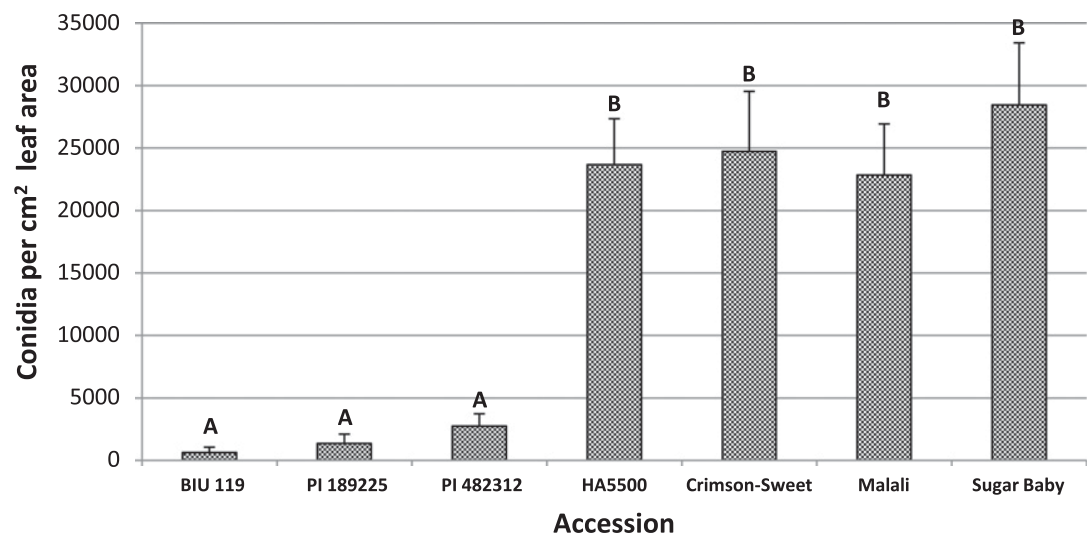

Fig. 2. Development of powdery mildew on seven accessions of Citrullus spp. Potted plants at their 4-leaf stage were inoculated with $\sim 150$ conidia/cm ${ }^{2}$ of Podospheara xanthii. A, Disease severity at 12 days postinoculation (dpi). B, Spore yield at 13 dpi. Different letters on bars indicate significant difference between means at $\alpha=0.05$. Pearson correlation coefficient between disease severity and spore yield is 0.90 . 
R/MR/S at a ratio of 1/43/20 (Table 5). Only 1 out of $64 \mathrm{~F} 2$ plants (9 resistant out of $525 \mathrm{~F} 2$ plants in three crosses) was fully resistant to powdery mildew, suggesting that three partially dominant complementary genes are responsible for resistance, regardless of the source of resistance.

The nine resistant plants (Table 5) were self-pollinated and their pedigree plants were all found to be resistant to powdery mildew race $1 \mathrm{~W}$ under field conditions in the following season.

Allelic relationships. To verify whether or not the three resistant accessions carry the same genes for resistance they were crossed with each other and their progeny plants tested for resistance at the cotyledon stage in the greenhouse and the 15-leaf stage under field conditions.

$\mathrm{F} 1, \mathrm{~F} 2$, and $\mathrm{BCs}$ progeny plants of all three crosses were resistant at the cotyledon stage (data not shown), suggesting that all accessions share the same partially dominant gene for resistance.

At the 15-leaf stage in the field, all offspring plants of the cross PI $189225 \times$ BIU 119 were resistant (Table 6 , cross A), suggesting that both parents carry the same resistance genes against $P$. xanthii. However, PI 482312 was only partially allelic for resistance with either PI 189225 (Table 6, cross B) or BIU 119 (Table 6, cross C) under field conditions. F1 and E1 (reciprocal cross) plants of cross B and cross $\mathrm{C}$ were moderately resistant, the F2 and E2 (reciprocal cross) progeny plants of both cross $B$ and cross $C$ segregated $R / M R$ at a ratio of $7 / 9$ and the $\mathrm{BC}$ offspring plants segregated 1/1R/MR, suggesting that PI 482312 shares two out of three genes with either PI 189225 or BIU 119.

Advanced breeding lines. BIU 119 was used to produce a resistant breeding line by crossing with the elite watermelon HA5500 as a recurrent parent. After a series of four BCs to HA5500, selection for resistance, and self-pollination, a highly resistant breeding line (TSS 11\%) was developed. The fruit shape, color, and resistance to powdery mildew race $1 \mathrm{~W}$ are shown in Figure 5.

\section{DISCUSSION}

The present study confirms that resistance against powdery mildew in watermelon could be transferred from wild resistant watermelon accessions into elite susceptible cultivars. The advanced breeding line we produced has high levels of resistance to powdery mildew and good fruit qualities (Fig. 5).

Of 291 watermelon accessions examined, only eight were highly resistant to powdery mildew race $1 \mathrm{~W}$. The resistant accessions PI 189225, PI 482323, and BIU 119 were crossed with susceptible parents to follow the mode of inheritance of resistance against the disease.

These accessions and their self-pollinated pedigree plants were resistant to the disease, showing no disease or reduced number of powdery mildew colonies on the hypocotyl, cotyledons, or the first true leaf. The mode of inheritance of resistance against race $1 \mathrm{~W}$ in all crosses was strongly dependent on plant organ and plant age at time of inoculation. When inoculated at the cotyledon stage, $25 \%$ of the F2 plants were resistant, $50 \%$ moderately resistant, and $25 \%$ susceptible, suggesting on a single, partially dominant gene controlling resistance. When inoculated at the 4-leaf stage, F2 plants segregated 7/37/20 $\mathrm{R} / \mathrm{MR} / \mathrm{S}, \mathrm{BCr}$ segregated $1 / 1 \mathrm{R} / \mathrm{MR}$, and $\mathrm{BCs}$ segregated $1 / 7$ $\mathrm{MR} / \mathrm{S}$. This indicated that three genes are responsible for resistance: full resistance is achieved by the complementary (additive) action of five or six partially dominant alleles. Plants showing intermediate resistance probably carry three or four dominant alleles (Table 7).

Of the $525 \mathrm{~F} 2$ plants inoculated (in all three crosses) at the 4-leaf stage, 59 plants were highly resistant. Nine of them remained highly resistant when transferred and grown in the field while the other 50 plants became moderately resistant, changing the segregation ratio to $1 / 43 / 20 \mathrm{R} / \mathrm{MR} / \mathrm{S}$. This suggests that resistance is controlled by the complementary/additive action of three partially dominant genes. In parents-offspring F2/F3 tests, F3 plants derived from the resistant F2 individuals were all resistant. We assume that when all six partially dominant alleles are present at the mature stage $(1 / 64$, Table 7$)$ reaction to disease will be highly resistant; three to five alleles (43/64) will mediate moderate resistance, and zero to two alleles (20/64) will contribute no resistance.

The allelic relationship between the three sources of resistance was examined by their intercrossings. BIU 119 and PI 189225 were fully allelic for resistance, although different in other traits (Table 2). PI 482312 shares two resistance genes with the other two accessions. We propose that BIU 119 and PI 189225 carry the genes $\mathrm{A}, \mathrm{B}$, and $\mathrm{C}$ for resistance whereas PI 482312 carry the resistance genes A, B, and D. Combining A, B, C, and D may enhance resistance against powdery mildew race $1 \mathrm{~W}$ to a maximal level.
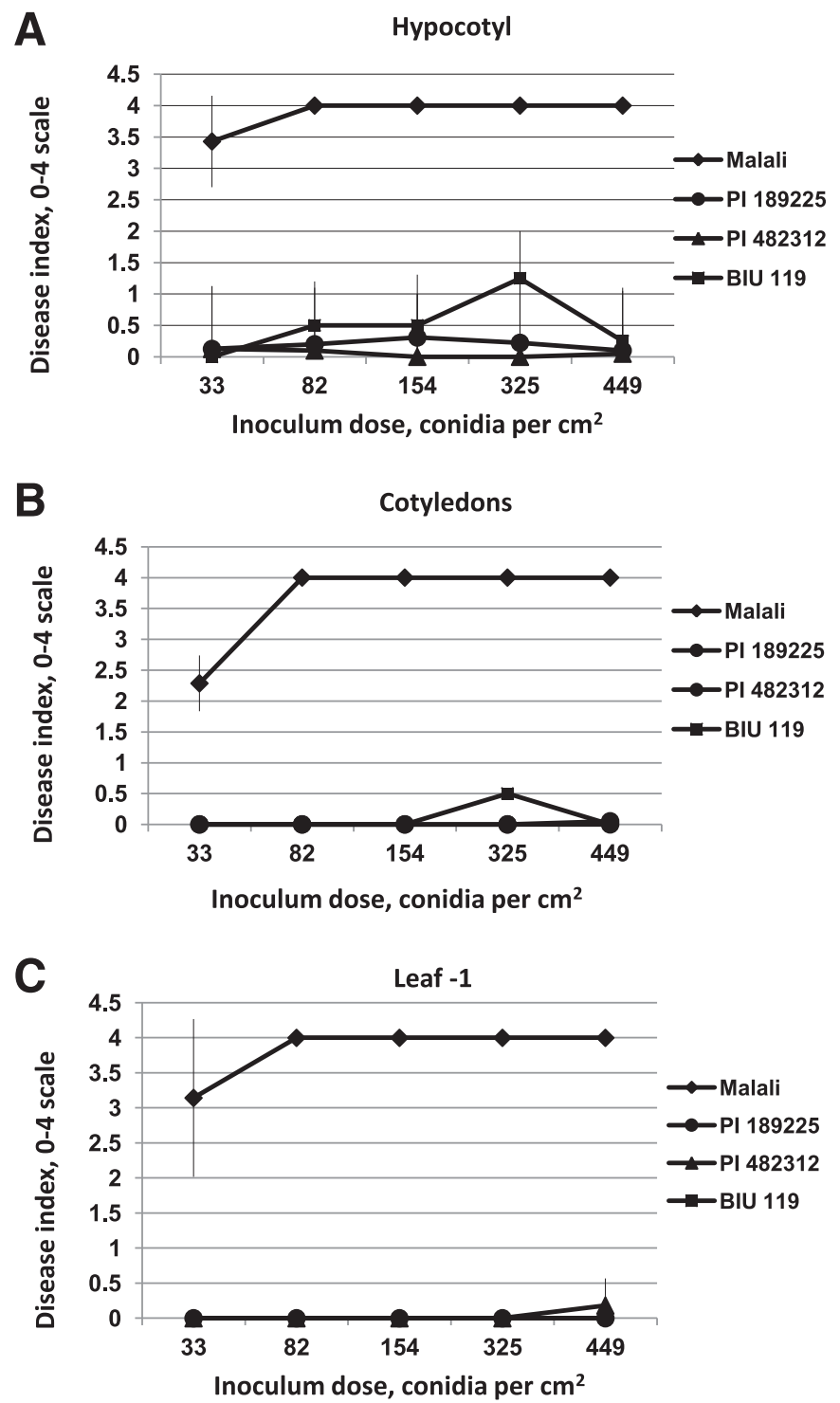

Fig. 3. The effect of inoculum dose on powdery mildew development in susceptible and resistant watermelon accessions. Plants at the first leaf stage were inoculated by overhead dusting of conidia of Podospheara xanthii at the indicated doses and evaluated for disease development at 10 days postinoculation. A, B, and C, Disease severity records taken from the hypocotyls, cotyledons, and leaf 1 , respectively. Bars indicate standard deviation of the mean. The susceptible cultivar Malali showed significantly $(\alpha=0.05)$ higher disease index values compared with all resistant accessions at all inoculum doses (analysis not shown). 
A
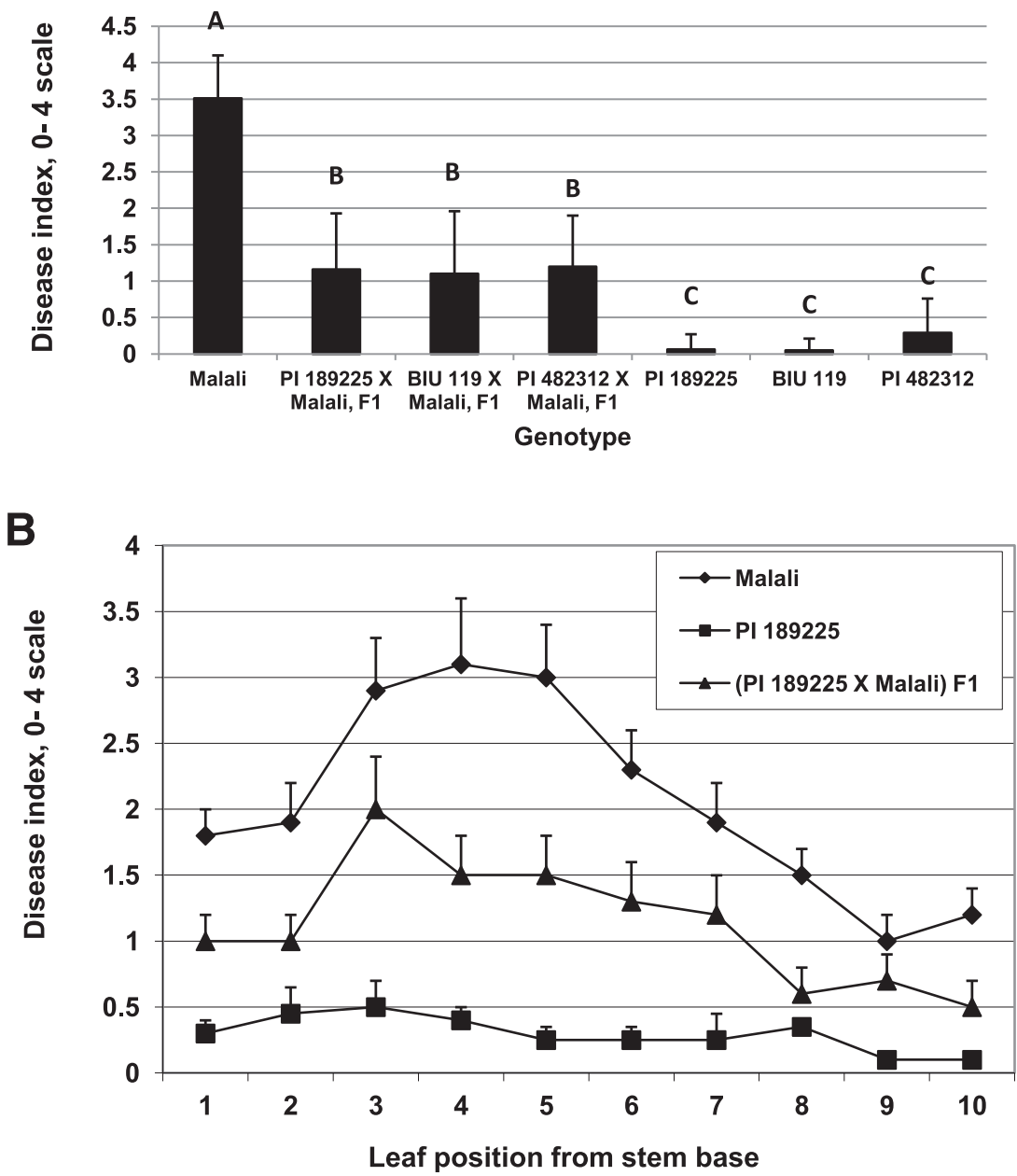

Fig. 4. A, Development of powdery mildew race $1 \mathrm{~W}$ on parents and F1 hybrid plants under field conditions. Different letters on bars indicate significant difference between means at $\alpha=0.05$. B, Effect of leaf age on development of powdery mildew in detached leaves of parental and F1 progeny plants. Leaves were detached from 10-leaf field-grown plants, placed on wet filter paper in transparent plastic plates $(20 \times 20 \times 3 \mathrm{~cm})$, dust-inoculated with conidia of Podospheara xanthii $\left(\sim 150\right.$ conidia per $\left.\mathrm{cm}^{2}\right)$, incubated in a growth chamber at $21^{\circ} \mathrm{C}, 12 \mathrm{~h}$ light/day, and evaluated for disease development at 10 days postinoculation. Different letters on bars indicate significant difference between means at $\alpha=0.05$.

TABLE 3. Inheritance of resistance to powdery mildew race $1 \mathrm{~W}$ in watermelon ${ }^{\mathrm{a}}$

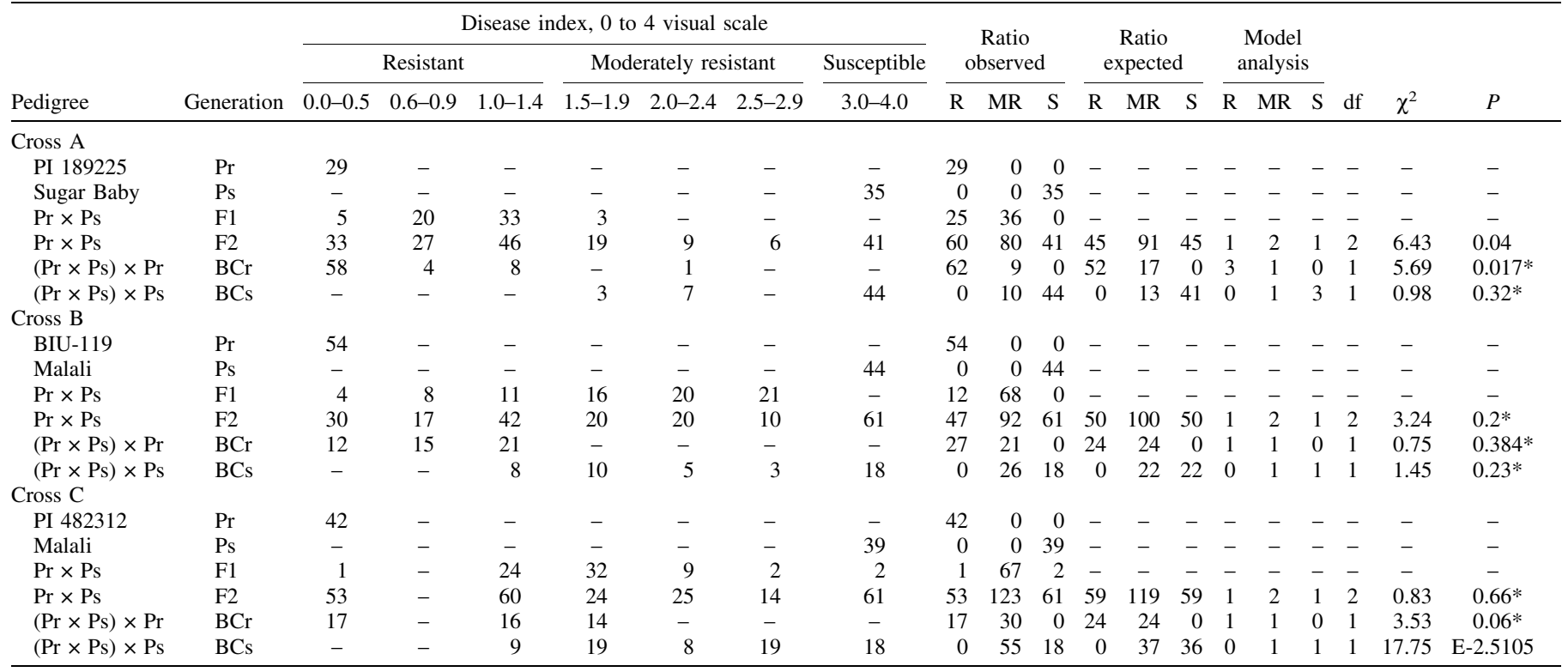

${ }^{a}$ Three resistant genotypes were crossed with the commercial susceptible Sugar Baby, and the cotyledons of the progeny plants were scored for resistance in the greenhouse. * Indicates model analysis accepted $(P \geq 0.05)$ in seven of nine cases. 
TABLE 4. Inheritance of resistance to powdery mildew race $1 \mathrm{~W}$ in watermelon ${ }^{\mathrm{a}}$

\begin{tabular}{|c|c|c|c|c|c|c|c|c|c|c|c|c|c|c|c|c|c|c|c|c|}
\hline \multirow[b]{3}{*}{ Pedigree } & \multirow[b]{3}{*}{ Generation } & \multicolumn{7}{|c|}{ Disease index, 0-4 visual scale } & \multirow{2}{*}{\multicolumn{3}{|c|}{$\begin{array}{c}\text { Ratio } \\
\text { observed }\end{array}$}} & \multirow{2}{*}{\multicolumn{3}{|c|}{$\begin{array}{c}\text { Ratio } \\
\text { expected }\end{array}$}} & \multirow{2}{*}{\multicolumn{3}{|c|}{$\begin{array}{c}\text { Model } \\
\text { analysis }\end{array}$}} & \multirow[b]{3}{*}{ df } & \multirow[b]{3}{*}{$\chi^{2}$} & \multirow[b]{3}{*}{$P$} \\
\hline & & \multicolumn{2}{|c|}{ Resistant } & \multicolumn{4}{|c|}{ Moderately resistant } & \multirow{2}{*}{$\frac{\text { Susceptible }}{3.0-4.0}$} & & & & & & & & & & & & \\
\hline & & $0.0-0.0 .5$ & $0.6-0.9$ & $1.0-1.4$ & $1.5-1.9$ & $2.0-2.4$ & $2.5-2.9$ & & $\mathrm{R}$ & MR & $\mathrm{S}$ & $\mathrm{R}$ & MR & $\mathrm{S}$ & $\mathrm{R}$ & MR & $\mathrm{S}$ & & & \\
\hline \multicolumn{21}{|l|}{ Cross A } \\
\hline PI 189225 & $\operatorname{Pr}$ & 31 & - & - & - & - & - & - & 31 & 0 & 0 & - & - & - & - & - & - & - & - & - \\
\hline Sugar Baby & Ps & - & - & - & - & - & - & - & 0 & 0 & 38 & - & - & - & - & - & - & - & - & - \\
\hline $\operatorname{Pr} \times \operatorname{Ps}$ & $\mathrm{F} 1$ & - & - & 4 & 22 & 21 & 10 & 7 & 0 & 57 & 7 & - & - & - & - & - & - & - & - & - \\
\hline $\operatorname{Pr} \times \operatorname{Ps}$ & $\mathrm{F} 2$ & 6 & 9 & 21 & 19 & 31 & 21 & 65 & 15 & 92 & 85 & 18 & 100 & 54 & 7 & 37 & 20 & 2 & 3.385 & $0.184 *$ \\
\hline$(\operatorname{Pr} \times \operatorname{Ps}) \times \operatorname{Pr}$ & $\mathrm{BCr}$ & 16 & 20 & 17 & 12 & 2 & - & - & 36 & 31 & 0 & 33 & 34 & 0 & 1 & 1 & 0 & 1 & 0.54 & $0.46^{*}$ \\
\hline$(\operatorname{Pr} \times \mathrm{Ps}) \times \mathrm{Ps}$ & $\mathrm{BCs}$ & - & - & - & - & 3 & 7 & 44 & 0 & 10 & 44 & 0 & 7 & 47 & 0 & 1 & 7 & 1 & 1.47 & $0.22 *$ \\
\hline \multicolumn{21}{|l|}{ Cross B } \\
\hline BIU 119 & $\operatorname{Pr}$ & 23 & - & - & - & - & - & - & 23 & 0 & 0 & - & - & - & - & - & - & - & - & - \\
\hline Malali & Ps & - & - & - & - & - & - & 21 & 0 & 0 & 25 & - & - & - & - & - & - & - & - & - \\
\hline $\operatorname{Pr} \times \operatorname{Ps}$ & $\mathrm{F} 1$ & - & - & 11 & 38 & 5 & 6 & 1 & 0 & 60 & 1 & - & - & - & - & - & - & - & - & - \\
\hline $\operatorname{Pr} \times \operatorname{Ps}$ & $\mathrm{F} 2$ & 11 & 10 & 25 & 19 & 10 & 17 & 41 & 21 & 72 & 41 & 15 & 78 & 41 & 7 & 37 & 20 & 2 & 2.85 & $0.24 *$ \\
\hline$(\operatorname{Pr} \times \operatorname{Ps}) \times \operatorname{Pr}$ & $\mathrm{BCr}$ & 37 & - & 26 & 1 & - & - & - & 37 & 26 & 0 & 32 & 32 & 0 & 1 & 1 & 0 & 1 & 1.9 & $0.168 *$ \\
\hline$(\operatorname{Pr} \times \operatorname{Ps}) \times \operatorname{Ps}$ & $\mathrm{BCs}$ & - & - & - & - & - & 2 & 38 & 0 & 2 & 38 & 0 & 5 & 35 & 0 & 1 & 7 & 1 & 2.06 & $0.151 *$ \\
\hline \multicolumn{21}{|l|}{ Cross $\mathrm{C}$} \\
\hline PI 482312 & $\operatorname{Pr}$ & 42 & - & - & - & - & - & - & 42 & 0 & 0 & - & - & - & - & - & - & - & - & - \\
\hline Malali & Ps & - & - & - & - & - & - & 39 & 0 & 0 & 39 & - & - & - & - & - & - & - & - & - \\
\hline $\operatorname{Pr} \times \operatorname{Ps}$ & $\mathrm{F} 1$ & - & - & 14 & 26 & 30 & 8 & 7 & 0 & 78 & 7 & - & - & - & - & - & - & - & - & - \\
\hline $\operatorname{Pr} \times \operatorname{Ps}$ & $\mathrm{F} 2$ & - & 23 & 27 & 41 & 42 & 21 & 65 & 23 & 131 & 65 & 24 & 126 & 69 & 7 & 37 & 20 & 2 & 0.47 & $0.79 *$ \\
\hline$(\operatorname{Pr} \times \operatorname{Ps}) \times \operatorname{Pr}$ & $\mathrm{BCr}$ & - & 28 & 16 & 4 & - & - & - & 28 & 20 & 0 & 24 & 24 & 0 & 1 & 1 & 0 & 1 & 0.065 & $0.8^{*}$ \\
\hline$(\mathrm{Pr} \times \mathrm{Ps}) \times \mathrm{Ps}$ & $\mathrm{BCs}$ & - & - & - & - & 2 & 18 & 50 & 0 & 20 & 50 & 0 & 9 & 61 & 0 & 1 & 7 & 1 & 3.17 & $0.075^{*}$ \\
\hline
\end{tabular}

${ }^{a}$ Three resistant genotypes were crossed with the susceptible commercial cultivars Sugar Baby or Malali and progeny plants were scored for resistance at the 4-leaf stage in the greenhouse. * Indicates model analysis accepted $(P \geq 0.05)$.

TABLE 5. Inheritance of resistance to powdery mildew race $1 \mathrm{~W}$ in watermelon ${ }^{\mathrm{a}}$

\begin{tabular}{|c|c|c|c|c|c|c|c|c|c|c|c|c|c|}
\hline \multirow[b]{2}{*}{ Cross } & \multirow[b]{2}{*}{ Generation } & \multicolumn{3}{|c|}{ Ratio observed } & \multicolumn{3}{|c|}{ Ratio tested } & \multicolumn{3}{|c|}{ Model analysis } & \multirow[b]{2}{*}{$\mathrm{df}$} & \multirow[b]{2}{*}{$\chi^{2}$} & \multirow[b]{2}{*}{$P$} \\
\hline & & $\mathrm{R}$ & MR & $\mathrm{S}$ & $\mathrm{R}$ & MR & $\mathrm{S}$ & $\mathrm{R}$ & MR & $S$ & & & \\
\hline PI $482312 \times$ Malali & F2 & 4 & 150 & 65 & 3 & 147 & 69 & 1 & 43 & 20 & 2 & 0.626 & $0.73 *$ \\
\hline BIU $110 \times$ Sugar Baby & F2 & 1 & 90 & 43 & 2 & 91 & 41 & 1 & 43 & 20 & 2 & 1.01 & $0.74 *$ \\
\hline
\end{tabular}

${ }^{a}$ Three resistant genotypes were crossed with the susceptible cultivars Malali or Sugar Baby. F2 progeny plants that were scored resistant or moderately-resistant at the 4-leaf stage in the greenhouse were transferred to the field and again scored for resistance at the 15-leaf stage. * Indicates model analysis accepted $(P \geq 0.05)$.

TABLE 6. The allelic relationships between three accessions of Citrullus spp., each carrying three partially dominant genes for resistance against powdery mildew race $1 \mathrm{~W}^{\mathrm{a}}$

\begin{tabular}{|c|c|c|c|c|c|c|c|c|c|c|c|c|c|c|}
\hline & \multirow[b]{2}{*}{ Generation } & \multicolumn{3}{|c|}{ Ratio observed } & \multicolumn{3}{|c|}{$\begin{array}{c}\text { Ratio } \\
\text { expected }\end{array}$} & \multicolumn{3}{|c|}{ Model analysis } & \multirow[b]{2}{*}{ df } & \multirow[b]{2}{*}{$\chi^{2}$} & \multirow[b]{2}{*}{$P$} & \multirow[b]{2}{*}{ Genes } \\
\hline & & $\mathrm{R}$ & MR & $\mathrm{S}$ & $\mathrm{R}$ & MR & $\bar{S}$ & $\mathrm{R}$ & MR & $\overline{\mathrm{S}}$ & & & & \\
\hline \multicolumn{15}{|l|}{ Cross A } \\
\hline BIU 119 & Pr-2 & 47 & 0 & 0 & 1 & 0 & 0 & 47 & 0 & 0 & - & - & - & \\
\hline BIU $119 \times$ PI 189225 & F1 & 66 & 0 & 0 & 1 & 0 & 0 & 66 & 0 & 0 & - & - & - & \\
\hline PI $189225 \times$ BIU 119 & E1 & 55 & 0 & 0 & 1 & 0 & 0 & 55 & 0 & 0 & - & - & - & \\
\hline \multicolumn{15}{|l|}{ Cross B } \\
\hline PI 189225 & $\operatorname{Pr}-1$ & 41 & 1 & 0 & - & - & - & - & - & - & - & - & - & Partially allelic \\
\hline PI 482312 & Pr-2 & 25 & 2 & 0 & - & - & - & - & - & - & - & - & - & \\
\hline PI $482312 \times$ PI 189225 & $\mathrm{~F} 1$ & 3 & 45 & 0 & - & - & - & - & - & - & - & - & - & \\
\hline PI 189225 × PI 482312 & E1 & 1 & 27 & 0 & - & - & - & - & - & - & - & - & - & \\
\hline BIU $119 \times 189225$ & $\mathrm{~F} 2+\mathrm{E} 2$ & 73 & 62 & 0 & 64 & 81 & 0 & 7 & 9 & 0 & 1 & 0.005 & 0.016 & \\
\hline PI $482312 \times$ BIU 119 & $\mathrm{~F} 1$ & 2 & 32 & 0 & - & - & - & - & - & - & - & - & - & \\
\hline BIU $119 \times$ PI 482312 & E1 & 6 & 48 & 0 & - & - & - & - & - & - & - & - & - & \\
\hline PI $189225 \times$ BIU 119 & $\mathrm{~F} 2+\mathrm{E} 2$ & 83 & 59 & 0 & 62 & 80 & 0 & 7 & 9 & 0 & 1 & $2.27 \mathrm{E}-07$ & 0.003 & \\
\hline$($ BIU $119 \times$ PI 482312$) \times$ PI 482312 & BC Pr-1 & 37 & 25 & 0 & 31 & 31 & 0 & 1 & 1 & 0 & 1 & 2.3 & $0.13 *$ & \\
\hline$($ BIU $119 \times$ PI 482312$) \times$ BIU 119 & BC Pr-2 & 66 & 49 & 0 & 58 & 57 & 0 & 1 & 1 & 0 & 1 & 2.3 & $0.14 *$ & \\
\hline
\end{tabular}




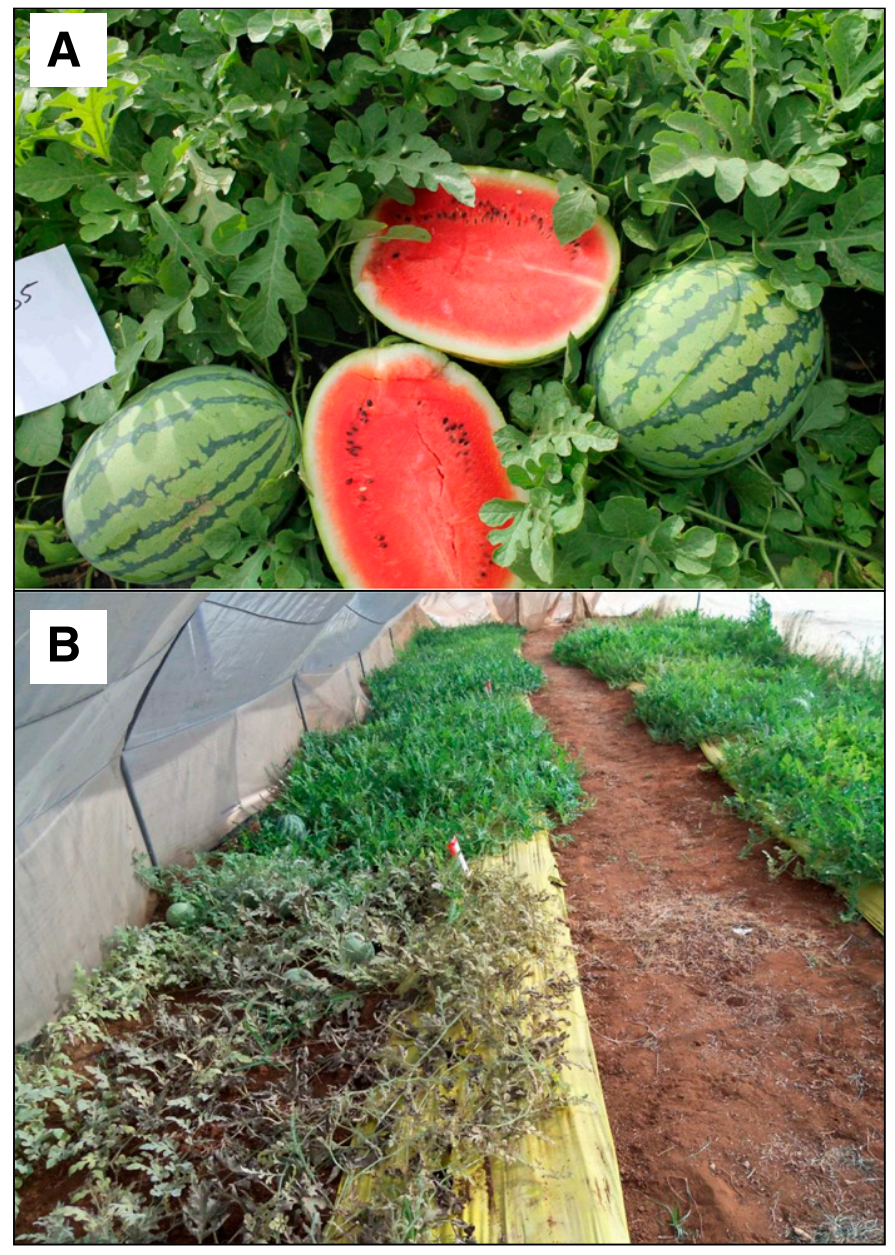

Fig. 5. A, Fruit shape, flesh color, and resistance to powdery mildew of an advance watermelon breeding line 35 developed in this study. Its pedigree is [((BIU $119 \times$ HA5500, F2) $\times$ HA5500)) self, BCs $\times 4$ ] . B, Response to powdery mildew race $1 \mathrm{~W}$ in a net-house in the field. Upper part: resistant line 35 ; lower part: susceptible cultivar HA5500.

TABLE 7. Segregation of partially dominant resistance alleles in a three-gene proposed model $^{\mathrm{a}}$

\begin{tabular}{|c|c|c|c|c|c|c|c|c|}
\hline Gametes & $A B C$ & $A B c$ & $\mathrm{AbC}$ & $A b c$ & $\mathrm{aBC}$ & $\mathrm{aBc}$ & $a b C$ & $a b c$ \\
\hline \multirow{2}{*}{$A B C$} & $A B C$ & $\mathrm{ABc}$ & $A b C$ & $\mathrm{Abc}$ & $\mathrm{aBC}$ & $\mathrm{aBc}$ & $a b c$ & $a b c$ \\
\hline & $\mathrm{ABC}$ & $\mathrm{ABC}$ & $A B C$ & $A B C$ & $A B C$ & $A B C$ & $A B C$ & $A B C$ \\
\hline \multirow{2}{*}{$\mathrm{ABC}$} & $A B C$ & $\mathrm{ABc}$ & $\mathrm{AbC}$ & $\mathrm{Abc}$ & $\mathrm{aBC}$ & $\mathrm{aBc}$ & $a b c$ & $a b c$ \\
\hline & $\mathrm{ABC}$ & $\mathrm{ABc}$ & $\mathrm{ABc}$ & $A B c$ & $\mathrm{ABC}$ & $\mathrm{ABC}$ & $\mathrm{ABc}$ & $\mathrm{ABC}$ \\
\hline \multirow{2}{*}{$\mathrm{AbC}$} & $\mathrm{ABC}$ & $\mathrm{ABc}$ & $\mathrm{AbC}$ & $\mathrm{Abc}$ & $\mathrm{aBC}$ & $\mathrm{aBc}$ & $a b c$ & $a b c$ \\
\hline & $\mathrm{AbC}$ & $A b C$ & $\mathrm{AbC}$ & $A b C$ & $A b C$ & $A b C$ & $A b C$ & $A b C$ \\
\hline \multirow{2}{*}{$A b c$} & $A B C$ & $\mathrm{ABc}$ & $A b C$ & $A b c$ & $\mathrm{aBC}$ & $\mathrm{aBc}$ & $a b c$ & $a b c$ \\
\hline & $A b c$ & $A b c$ & $A b c$ & $A b c$ & $A b c$ & $A b c$ & $A b c$ & $\mathrm{Abc}$ \\
\hline \multirow{2}{*}{$\mathrm{aBC}$} & $A B C$ & $\mathrm{ABc}$ & $A b C$ & $\mathrm{Abc}$ & $\mathrm{aBC}$ & $\mathrm{aBc}$ & $a b c$ & $a b c$ \\
\hline & $\mathrm{aBC}$ & $\mathrm{aBC}$ & $\mathrm{aBC}$ & $\mathrm{aBC}$ & $\mathrm{aBC}$ & $\mathrm{aBC}$ & $\mathrm{aBC}$ & $\mathrm{aBC}$ \\
\hline \multirow{2}{*}{$\mathrm{aBc}$} & $A B C$ & $\mathrm{ABc}$ & $\mathrm{AbC}$ & $A b c$ & $\mathrm{aBC}$ & $\mathrm{aBc}$ & $a b c$ & $a b c$ \\
\hline & $\mathrm{aBc}$ & $\mathrm{aBc}$ & $\mathrm{aBc}$ & $\mathrm{aBc}$ & $\mathrm{aBc}$ & $\mathrm{aBc}$ & $\mathrm{aBc}$ & $\mathrm{aBc}$ \\
\hline \multirow{2}{*}{$a b c$} & $A B C$ & $\mathrm{ABc}$ & $\mathrm{AbC}$ & $A b c$ & $\mathrm{aBC}$ & $\mathrm{aBc}$ & $a b c$ & $a b c$ \\
\hline & $a b C$ & $a b c$ & $a b C$ & $a b C$ & $a b C$ & $a b C$ & $a b c$ & $a b c$ \\
\hline \multirow{2}{*}{$a b c$} & $\mathrm{ABC}$ & $\mathrm{ABc}$ & $\mathrm{AbC}$ & $\mathrm{Abc}$ & $\mathrm{aBC}$ & $\mathrm{aBc}$ & $a b c$ & $a b c$ \\
\hline & $a b c$ & $a b c$ & $a b c$ & $a b c$ & $a b c$ & $a b c$ & $a b c$ & $a b c$ \\
\hline
\end{tabular}

a The complementary action of six alleles is required for full resistance of watermelon against Podosheara xanthii race 1W. Lower levels of resistance are obtained by the complementary action of three to five alleles. The complementary action of one to two alleles contributes no visible resistance. All three resistant accessions share the resistance genes A and B. The third resistance gene in accessions BIU 119 and PI 189225 is C, whereas the third resistance gene in accession PI 482312 is D (not shown in the Table).

\section{LITERATURE CITED}

Cohen, Y., Baider, A., Petrov, L., Sheck, L., and Voloisky, V. 2000. Crossinfectivity of Sphaerotheca fuliginea to watermelon, melon and cucumber. Proceedings of 7th EUCARPIA Meeting on Cucurbit Genetics and Breeding. N. Katzir and H. S. Paris, eds. Acta Hortic. 510:85-88.

Cohen, Y., and Eyal, H. 1995. Differential expression of resistance to powdery mildew incited by race 1 or 2 of Sphaerotheca fuliginea in Cucumis melo genotypes at various stages of plant development. Phytoparasitica 23:223-230

Davis, A. R., Bruton, B. D., Pair, S. D., and Thomas, C. E. 2001. Powdery mildew: An emerging disease of watermelon in the United States. Cucurbit Genet. Coop. 24:42-48.

Davis, A. R., Levi, A., Tetteh, A., Wehner, T., Russo, V., and Pitrat, M. 2007. Evaluation of watermelon and related species for resistance to race $1 \mathrm{~W}$ powdery mildew. J. Am. Soc. Hortic. Sci. 132:790-795.

Davis, A. R., Levi, A., Wehner, T., and Pitrat, M. 2006a. PI525088-PMR, a melon race 1 powdery mildew-resistant watermelon line. HortScience 41: $1527-1528$.

Davis, A. R., Tetteh, A., Wehner, T., Levi, A., and Pitrat, M. 2006 b. Watermelon resistance to powdery mildew race 1 and race 2. Pages 412-420 in: Proc. Cucurbitaceae. G. J. Holmes, ed. Universal Press, Raleigh, NC.

Davis, A. R., Thomas, C. E., Levi, A., Bruton, B. D., and Pair, S. D. 2002. Watermelon resistance to powdery mildew race 1 . Cucurbitaceae 2 : 192-198.

Felix-Gastelum, R., Apodaca-Sanchez, M. A., and Martinez-Valenzuela, M. C. 2005. Podosphaera (sect. Sphaerotheca) xanthii (Castagne) U. Brawn and N. Shishkoff on cucurbits in northern Sinaloa, Mexico. Rev. Mex. Fitopatol. 23:162-168.

Keinath, A. P., and DuBose, B. 2004. Evaluation of fungicides for prevention and management of powdery mildew on watermelon. Crop Prot. 23:35-42.

Kenigsbuch, D., and Cohen, Y. 1992. Inheritance and allelism of genes for resistance against races 1 and 2 of Sphaerotheca fuliginea in muskmelons. Plant Dis. 76:626-629.

Kim, K. H., Ahn, S. G., Hwang, J. H., Choi, Y. M., Moon, H. S., and Park, Y. H. 2013. Inheritance of resistance to powdery mildew in the watermelon and development of a molecular marker for selecting resistant plants. Hortic. Environ. Biotechnol. 54:134-140.

Kousik, C. S., Donahoo, R. S., Webster, C. G., Turechek, W. W., Adkins, S. T., and Roberts, P. D. 2011. Outbreak of cucurbit powdery mildew on watermelon fruit caused by Podosphaera xanthii in Southwest Florida. Plant Dis. 95:1586.

Lebeda, A., Kristkova, E., and Sedlakova, B. 2011. Gaps and perspectives of pathotype and race determination in Golovinomyces cichoracearum and Podosphaera xanthii. Mycoscience 52:159-164.

Levi, A., Harris, K. R., Wechter, W. P., Kousik, C. S., and Thies, J. A. 2010. DNA markers and pollen morphology reveal that Praecitrullus fistulosus is more closely related to Benincasa hispida than to Citrullus spp. Genet. Resour. Crop Evol. 57:1191-1205.

Levi, A., Thomas, C. E., Simmons, A. M., and Thies, J. A. 2005. Analysis based on RAPD and ISSR markers reveals closer similarities among Citrullus and Cucumis species than with Praecitrullus fistulosus (Stocks) Pangalo. Genet. Resour. Crop Evol. 52:465-472.

McCreight, J. D., Pitrat, M., Thomas, C. E., Kishaba, A. N., and Bohn, G. W. 1987. Powdery mildew resistance genes in muskmelon. J. Am. Soc. Hortic. Sci. 112:156-160.

McGrath, M. T. 2001. Distribution of cucurbit powdery mildew races 1 and 2 on watermelon and muskmelon. Abstr. Phytopathology 91(suppl.): 197.

McGrath, M. T., and Thomas, C. E. 1996. Powdery mildew. Pages 28-30 in: Compendium of Cucurbit Diseases. T. A. Zitter, D. L. Hopkins, and C. E. Thomas, eds. American Phytopathological Society, St. Paul, MN.

Mercier, J., Muscara, M. J., and Davis, A. R. 2014. First Report of Podosphaera xanthii race $1 \mathrm{~W}$ causing powdery mildew of watermelon in California. Plant Dis. 98:158.

Pitrat, M., Dogimont, C., and Bardin, M. 1998. Resistance to fungal diseases of foliage in melon. Pages 167-173 in: Cucurbitaceae '98: Evaluation and Enhancement of Cucurbit Germplasm. J. D. McCreight, ed. ASHS Press, Alexandria, VA.

Tetteh, A. Y., Wehner, T. C., and Davis, A. R. 2010. Identifying resistance to powdery mildew race $2 \mathrm{~W}$ in the USDA-ARS watermelon germplasm collection. Crop Sci. 50:933-939.

Tetteh, A. Y., Wehner, T. C., and Davis, A. R. 2013a. Inheritance of resistance to powdery mildew race 2 in Citrullus lanatus var. lanatus. HortScience 48: 1227-1230.

Tetteh, A. Y., Wehner, T. C., and Davis, A. R. 2013b. Inheritance of resistance to the new race of powdery mildew in watermelon. Crop Sci. 53: 880-887. 
Thomas, C. E., Levi, A., and Caniglia, E. 2005. Evaluation of US plant introductions of watermelon for resistance to powdery mildew. HortScience 40:154-156.

Tomason, Y., and Gibson, P. T. 2006. Fungal characteristics and varietal reactions of powdery mildew species on cucurbits in the steppes of Ukraine. Agron. Res. 4:549-562.
Zhang, H., Guo, S., Gong, G., Ren, Y., Davis, A. R., and Xu, Y. 2011. Sources of resistance to race $2 \mathrm{WF}$ powdery mildew in US watermelon plant introductions. HortScience 46:1349-1352.

Zohary, D., Hopf, M., and Weiss, E. 2012. Domestication of Plants in the Old World. Oxford University Press, New York. 